\title{
Dieter Mans
}

\author{
Soziologische Kausalanalyse \\ Eine Betrachtung aus großer Distanz
}

\begin{abstract}
Is causal analysis an appropriate method to discover causal laws? Some prerequisites for any successful application of mathematical causal models are worked out and discussed. It can be shown that these prerequisites cannot be fulfilled by present models and there are strong reasons to assume that they cannot be satisfied by any model. Therefore causal analysis will probably be of no importance for the discovery of causal laws in sociology.
\end{abstract}

"Den genauen Beginn der Seuche - wenn man überhaupt von einer Seuche reden sollte - kann niemand datieren. Die ersten Berichte wurden Anfang 1985 publiziert. In ihnen wurden Menschen beschrieben, die schlicht außerstande waren, ein naturwissenschaftliches Verständnis zu entwickeln, das über den Stand des späten IIten Jahrhunderts hinausging. Was ihnen fehlte war die Fähigkeit zu kontrollierten Experimenten und dementsprechend die Farhigkeit, neues Wissen zu gewinnen oder vorhandenes experimentell nachzuvollziehen.

Diese Beschreibungen wurden zuerst nicht ernst genommen, ja sie konnten gar nicht ernst genomren werden, dazu war das Phänomen viel zu kurios. Entweder hielt man das ganze für den spinnerten Einfall publicitysüchtiger Journalisten oder man glaubte, die beschriebenen Fälle seien einfach durch die Dummheit oder Bösartigkeit der Betroffenen zu erklären. Doch meistens nahm man die Berichte gar nicht zur Kenntnis. Was sollte man auch mit Berichten anfangen, wo das Verhalten von Erwachsenen beschrieben wurde, die sich tollpatschig wie Clowns oder Kinder verhielten, obwohl sie vorgaben, sich allen Ernstes um die Beherrschung einfacher technischer Geräte zu bemühen?

Das änderte sich erst Jahre später, als die Zahl der Fälle dramatisch zunahm und einer zunehmend hysterischen Öffentlichkeit die Schwere der Bedrohung deutlich wurde. Denn die Betroffenen (sollen wir sagen 'die Erkrankten'?) waren weder dumm noch bösartig, sie unterschieden sich in nichts von ihren Zeitgenossen, abgesehen von dem geschilderten Symptor: $\mathrm{Zu}$ kontrollierten Experimenten - auBer solchen einfachster Art - waren sie nicht imstande. Es gab unter ihnen brillante Theoretiker, die aber ohne die Unterstützung von 'Gesunden' hilflos waren, was die Überprüfung ihrer Theorien anging, so da $B$ sie sich bald in immer irrelevanteren Spekulationen ergingen.

Solange die Zahl der von der Seuche Betroffenen relativ gering war, hoffte man die Schwierigkeiten durch Arbeitsteilung zu bewältigen, aber diese Absicht erwies sich mit umfassenderer Ausbreitung als nicht durchführbar. Bald war die Zahl der nicht Erkrankten so gering, daß durch sie allein die technisch-wissenschaftliche Kultur nicht bewahrt werden konnte. Das war 
auch um so schwieriger, als die Identifikation eines Gesunden durch den damit verbundenen Zugewinn an sozialer Macht sehr problematisch war. Es gab keine einfachen Kriterien, nach denen man sie erkennen konnte, schon gar nicht für die von der Krankheit befallenen, so daß alle Arten von Scharlatanerie aufblühten.

$\mathrm{Da}$ der Niedergang der technisch-wissenschaftlichen Kultur nirgendwo gestoppt werden konnte, wurden Berichte über angeblich noch existierende Gesunde immer unglaubwürdiger und schließlich von niemandem mehr ernst genommen. Man schien weitgehend die Unabwendbarkeit der Entwicklung zu akzeptieren und verfolgte zugleich mit äuBerster Schärfe alle Arten von Scharlatanerie, da aus durchsichtigen Motiven immer phantastischere Möglichkeiten erörtert wurden, dem Unvermeidlichen zu entrinnen und an die Leistungen der nun über so Jahre vergangenen Epoche anzuknüpfen und sie fortzuführen.

Ob dieses harte Durchgreifen berechtigt war, bezweifle ich, denn durch die spektakulären Prozesse bekamen die Scharlatane erst jene Aufmerksamkeit, die ihnen sonst vermutlich nie zuteil geworden wäre. Zwar gab es wirklich nicht einen einzigen Beweis dafür, daß man an die technisch naturwissenschaftlichen Leistungen der früheren Zeiten anders als durch die unzugänglich gewordenen For schungsmethoden anknüpfen könnte, aber zumindest in einem Fall kam es zu einem spektakulären ProzeB wegen Scharlatanerie, bei dem ich mir nicht sicher bin, ob nicht eine hoffnungsvolle Forschungsrichtung eliminiert wurde.

Angeklagt waren Soziologen, genauer Methodologen, die behaupteten, es gäbe nicht-experimentelle Möglichkeiten kausale Gesetzmäßigkeiten zu finden. Ihre Argumentation beruhte auf einer in den 6oer Jahren des 20. Jahrhunderts populären Forschungsrichtung 'causal modeling' genannt, mit der der Anspruch auf eine nicht-experimentelle Kausalforschung eingelöst werden sollte. Die grundlegende Idee dieser Verfahren wurde so erläutert: Man erhebt Daten von einem bestimmten Zeitpunkt, zumeist Umfragedaten, wie sie z.B. in der Soziologie gängig waren, und versucht dann durch bestimmte Modellannahmen und Rechenverfahren Rückschlüsse auf die kausalen Zusammenhänge zwischen den erhobenen Variablen zu ziehen ..."

\section{Soziologische Kausalanalyse - eine Methode nur für Soziologen?}

Wir wollen den Proze $B$ und seinen für die angeklagten Soziologen so unerfreulichen Ausgang nicht weiter verfolgen, sondern gleichsam als imaginäre Beisitzer prüfen, ob es gute Gründe für ihre Verurteilung gegeben hat oder ob tatsächlich, wie unser Chronist meint, eine hoffnungsvolle Forschungsrichtung eliminiert wurde. Aber selbst zu dieser Frage können wir nur indirekt beitragen, da wir nur klären wollen, ob soziologisch einschlägige Kausalanalyseverfahren wenigstens in ihrem ureigensten Bereich eine Hilfe bei der Auffindung kausaler Regelmäßigkeiten sind. Wenn sie dies nicht sind - und dafür versuchen wir in der vorliegenden Arbeit einige Argumente zusammenzutragen -, dann werden sie es a fortiori auch nicht in naturwissenschaftlichen Verwendungszusammenhängen sein. Dafür werden wir uns nicht um weitere Argumente bemühen, da die Arbeit eher an Methodologen des Jahres 1984 als an ein Schwurgericht des Jahres 2043 gerichtet ist. Wir werden demnach allenfalls am Rande auf den Vergleich naturwissenschaftlicher und sozialwissenschaftlicher Forschungsmethoden ein- 
gehen, und dies allein, um unsere Kritik an soziologischen Kausalanalyseverfahren (angewandt auf soziologische Probleme) deutlich zu machen.

Trotzdem erscheint vor der Beschäftigung mit dem eigentlichen Thema der Arbeit ein kleiner verwunderter Blick auf typische Nichtanwendungen soziologischer Kausalanalyseverfahren hilfreich zu sein. Mir ist kein einziger Fall bekannt, in dem soziologische Kausalanalyseverfahren angewandt wurden, um naturwissen schaftliche Gesetzmäßigkeiten aufzudecken. Und selbst wenn es einzelne isolierte Anwendungen geben sollte: Gewöhnlich werden sie in den naturwissenschaftlichen Forschungsprozessen nicht benötigt und eine Übernahme als Lehrstoff in naturwissenschaftliche Curricula ist nicht zu erwarten.

So etwas nur zu denken erscheint eigenartig und weltfremd und von jeder Forschungsrealität abgehoben. Zu einfach sind die Argumente, mit denen die wechselseitige Nichtbeachtung begründet werden kann. Naturwissenschaftler arbeiten entweder an Problemen, die experimentellen Methoden zugänglich sind oder an Problemen (z.B. Himmelsmechanik, Meteorologie), deren Lösung auf verwickelte Weise auf ein experimentell erworbenes Wissen mit zurückgreift. Soziologen dagegen können (und dürfen) nur selten systematisch experimentieren, so da B sie für die Gewinnung kausaler Zusammenhänge andere Methoden verwenden müssen. Deswegen wäre es einfach verfehlt, Methoden aus dem einen Bereich in den anderen übertragen zu wollen.

Aber mit einer solchen Argumentation mogelt man sich an dem Problem vorbei, indem man aus den vorhandenen Unterschieden illegitime Schlußfolgerungen zieht. Denn die Frage ist doch, warum man in den Naturwissenschaften mit experimentellen Methoden arbeiten soll, wenn es eine methodisch weitaus einfachere Alternative gibt, wie sie durch die kausale Modellbildung der Soziologie scheinbar angeboten wird. Hierauf kann man nicht antworten, die Gegenstandsbereiche seien unterschiedlich und erforderten unterschiedliche Methoden. Die Hydrodynamik und die Kernphysik sind auch unterschiedliche Gegenstandsbereiche, die aber gleichwohl in einem wichtigen methodischen Punkt in gleicher Weise vorgehen: Sie überprüfen die Richtigkeit ihrer Theorien (auch) durch kontrollierte Experimente.

Aus der Tatsache der unterschiedlichen Gegenstandsbereiche lassen sich demnach keine Argumente gewinnen. Denn entweder haben beide Disziplinen das gleiche Ziel, nämlich die Entdeckung kausaler Zusammenhänge, dann sollten sie auch die gleichen Methoden verwenden, oder sie verfolgen unterschiedliche Zielsetzungen, dann sollte man deutlich machen, worin die Differenzen bestehen und gegebenenfalls darauf verzichten, in der Soziologie von Kausalanalyse zu reden.

Worin könnten die Unterschiede bestehen, die eine Verwendung unterschiedlicher Methoden rechtfertigen? Zumindest vier Strategien der Argumentation sind zur Rechtfertigung denkbar: 
(i) Naturwissenschaftler und Soziologen haben es mit unterschiedlichen Arten von Kausalität zu tun;

(ii) in der Soziologie gibt es gar keine kausalen Gesetze;

(iii) naturwissenschaftliche Gesetze genügen nicht den Voraussetzungen, die wir bei Anwendung soziologischer Analysemethoden machen müssen, etwa Linearität, Additivität, Normalverteilungsannahmen etc.;

(iv) im Prinzip liegt die gleiche Problemstellung vor, aber wegen der Unmöglichkeit in der Soziologie in größerem Umfang zu experimentieren, muß es eine Möglichkeit nicht-experimenteller Kausalanalyse geben, die dann aber nicht so gut sein kann, wie die experimentelle Methode.

Selbst wenn (i) korrekt wäre, könnte es nur dann als Argument dienen, wenn damit der Unterschied in den Analysetechniken begründet werden könnte. Da hierfür in der Literatur nicht ein einziges Argument vorhanden ist und auch schwer vorstellbar ist, worin ein solches Argument bestehen könnte, braucht man diese Möglichkeit nicht weiter ernst zu nehmen. Denn entscheidend für die experimentelle Erforschung kausaler Gesetzmäßigkeit ist, $\underline{\mathrm{da} B}$ bestimmte Ereignisse andere Ereignisse nach sich ziehen und nicht, welche internen Mechanismen dafür verantwortlich sind. Worin die Natur kausaler Verknüpfungen (z.B. im Gegensatz zu logischen und kontingenten) besteht, ist eine alte philosophische Streitfrage, die die konkrete Forschungspraxis unberührt läßt. Selbst der philosophisch dramatische Übergang von deterministischer zu probabilistischer Kausalität und die noch darüber hinausgehende Vermutung, die (starke) Kausalbeziehung sei in der modernen Physik nur von geringer Bedeutung, haben die Art experimenteller Forschung nicht verändert.

Wegen des Mangels an guten Argumenten für (i) brauchen wir uns mit dieser Art von Begründung nicht weiter auseinanderzusetzen. Es ist aber trotzdem wichtig zu sehen, warum aus dieser Ecke allenfalls philosophisch aber nicht forschungspraktisch bedeutsame Argumente kommen können.

Die Bewertung von (ii) ist ähnlich, wenn auch die Art der Begründung anders ist. Man kann es sich ganz einfach machen, indem man die Begründungslast umkehrt. Denn wenn es in der Soziologie keine Kausalität gibt, dann kann es auch keine Anwendungen kausal-analytischer Analyseverfahren geben. Das aber impliziert eine wesentlich schärfere Kritik an der Verwendung dieser Verfahren als sie hier vorgetragen wird, weil dann die Verwendung schlicht unsinnig ist.

Man kann (ii) auch in einer interessanteren Form vortragen, indem man an die 'reasons and causes'-Debatte anknüpft. Eine der vielen dort vertretenen Positionen impliziert, daß die Verknüpfung zwischen den Gründen einer 
Handlung und ihrer Ausführung stärker ist als kausal (konzeptuell oder logisch). Folgt man diesem Argumentationsstrang, dann ergeben sich wichtige Fragen zur Natur sozialwissenschaftlicher Forschungsprozesse, aber man landet schließlich bei Positionen, die eine kausale Analyse nicht zulassen. Das Argument (ii) ist demnach ungeeignet, um die Unterschiede zwischen naturwissenschaftlicher und soziologischer kausalerklärender Vorgehensweise zu begründen. Wer es verwendet, schüttet gleichsam das Kind mit dem Bade aus, denn er liefert kein Argument für die Verwendung nicht-experimenteller kausalanalytischer Verfahren, sondern eines, das die Verwendung solcher Verfahren generell problematisiert.

(iii) ist deswegen ein potentiell akzeptables Argument, weil es die Anwendung unterschiedlicher Kausalanalyseverfahren auf Unterschiede im Gegenstandsbereich zurückführt. Das überzeugendste Argument, um (iii) zu stützen, wäre

- eine erfolgreiche Anwendung soziologischer Analyseverfahren zur Entdeckung sozialer Gesetzmäßigkeiten;

- ein sich daran anschließender Vergleich zwischen der Struktur zweier Gegenstandsbereiche, der die Unterschiede verdeutlichen könnte, die eine Anwendung der Methoden des jeweils anderen Bereichs verhindern.

Nur fehlt es leider an guten Argumenten für die Begründung von (iii), weil es weder erfolgreiche Anwendungen soziologischer Kausalanalyseverfahren in ihrem eigenen Gegenstandsbereich gibt, noch verfügen wir über Argumente, warum physikalische Anwendungen grundsätzlich nicht den in der Soziologie üblichen Annahmen genügen. Der zweite Einwand gilt um so mehr, als wir nicht bei allen Kausalanalyseverfahren derartige Voraussetzungen machen müssen. Zwar dürfte es nicht schwierig sein, in Modellstudien nachzuweisen, $\mathrm{da} B$ es aussichtslos ist, mit soziologischen Kausalanalyseverfahren auf nicht experimentellem Wege naturwissenschaftliche Gesetze zu finden, nur zeigt das daraus folgende Argument gleichsam in die falsche Richtung: Solche Fehlanwendungen problematisieren diese Verfahren in ihrer Eigenschaft als Kausalanalyseverfahren. Nur wenn sie in der Soziologie mit Erfolg appliziert würden, könnte man die Nichtanwendung in den Naturwissenschaften auf strukturelle Unterschiede in den Gegenstandsbereichen zurückführen.

Aber davon kann keine Rede sein und es wäre auch ein extremer Zufall, wenn mehr oder weniger kontingent entwickelte und angewandte Methoden zu den tatsächlichen Gegebenheiten so paßten wie der Schlüssel zum Schloß. Unter "kontingent" soll hier der Umstand verstanden werden, da B solche Methoden nicht in einer sensiblen Auseinandersetzung mit inhaltlichen soziologischen Fragestellungen entwickelt wurden, sondern teils aus anderen Wissenschaftszweigen adaptiert oder einfach nach statistischen Möglichkeiten maßgeschneidert wurden. Gegen eine solche Vorgehensweise 
gibt es nichts einzuwenden, solange man nicht die hier diskutierte These vertritt, die soziologischen Kausalanalysemethoden paßten nur zu einem Gegenstandsbereich. Bislang jedenfalls fehlt ein überzeugender Nachweis, da $B$ gerade die soziale Realität mit den in ihr geltenden Kausalgesetzen für die gewählte Form der Analyse besonders geeignet sei. Alles in allem ist also (iii) ebenso unbefriedigend wie (i) oder (ii) und kann daher den Unterschied in den Forschungsmethoden nicht erklären.

Was bleibt ist die vierte Deutung, die vor allen anderen den Vorzug der Redlichkeit hat. Man versucht erst gar nicht, durch Hinweis auf Unterschiede in Gegenstandsbereichen die Differenzen zwischen naturwissenschaftlicher und sozialwissenschaftlicher Kausalanalyse zu erklären. Man sagt stattdessen: "Was wir wollen ist eine Art umfassender kausaler Theorie des Sozialen, aber wir können die Forschung nicht im naturwissenschaftlichen Stil aufziehen. Deswegen müssen wir uns mit prinzipiell schlechteren Methoden bescheiden, um wenigstens soviel wie irgend möglich herauszufinden." Vermutlich würde kein Soziologe (jedenfalls kein mir bekannter, der über kausale Analyse schreibt) so weit gehen und die Schaffung einer Physik des Sozialen als das Ziel soziologischer Kausalanalyse ausgeben. Aber wenn man mit Methoden arbeitet, die der Entdeckung von kausalen Gesetzmäßigkeiten dienen, dann muß man auch die Idee eines von kausalen Gesetzen strukturierten Gegenstandsbereichs akzeptieren und damit dem Gesetzbegriff die Priorität einräumen, die er in den Naturwissenschaften hat.

Ich formuliere dies bewuBt so provokativ in einer Sprache, wie sie von Sozialwissenschaftlern selten verwendet wird, weil ich so plastisch wie irgend möglich auf den Umstand hinweisen möchte, daß die Verwendung von typisch soziologischen Analysemethoden - i.e. der weitgehende Verzicht auf kontrollierbare Experimente - ein Schwachpunkt der soziologischen Kausalmethodologie ist, den man auch als Schwachpunkt identifizieren muB, um die Defizite der Analy seform zu erkennen. Man darf die (nichtexperimentellen) Verfahren der Soziologie nicht als den Versuch verstehen, grundsätzlich andere Fragestellungen mit grundsätzlich anderen Methoden zu behandeln, weil sich dann die Defizite der nichtexperimentellen Verfahren im Dunst methodologischer Unklarheit verlieren, bis man schließlich glaubt, gute analytische Instrumente zur Hand zu haben.

Wenn Formulierungen wie "Naturwissenschaft des Sozialen" provokativ wirken, dann sicher auch deswegen, weil selbst die empirischen Sozialforscher, die von kausalen Analysemethoden einen massiven Gebrauch machen, ihrem Selbstverständnis nach keine Naturwissenschaftler des Sozialen sind. Aber neben dem Selbstverständnis der Praktiker gibt es die Implikationen aus den verwendeten Verfahren und dort scheinen mir Zweifel an den damit übernommenen Zielsetzungen unangebracht zu sein: Wer kausale Analy- 
se betreibt, kann nicht verschämt so tun, als ginge es ihm gar nicht um eine Modellierung des Gegenstandsbereichs unter kausalen Gesetzen, sondern um etwas schwächeres, etwas viel harmloseres. Hier gilt eine Art methodologisches 'Mitgegangen - Mitgehangen', sprich: wer einen Gegenstandsbereich nach dem Schema der Kausalität konzeptualisiert, der setzt sich auch einer entsprechenden Kritik aus, wenn seine Methoden nicht greifen oder vielleicht gar nicht greifen können.

Um an dieser Stelle keine Mißverständnisse aufkommen zu lassen, sollten wir deutlich machen, daß uns zwei Fragen nicht interessieren, nämlich

- ist die soziale Realität tatsächlich durch kausale Gesetzmäßigkeiten systematisierbar?

- und wenn sie durch kausale GesetzmäBigkeiten systematisierbar ist, ist es dann fair, vorliegende theoretische Ansätze an dem Beispiel erfolgreicher naturwissenschaftlicher Theorien zu messen? Sind denn m.a.W. die Probleme trotz der Berufung auf Kausalität nicht grundsätzlich anders?

Obwohl die Antwort auf die erste Frage vermutlich 'nein' ist, braucht sie uns nicht zu interessieren. Die Problemstellung (kann man mit den diskutierten Methoden kausale Gesetzmäßigkeiten entdecken?) wird uns durch die Verwendung der Methoden vorgegeben. Wir müssen nicht an eine Physik des Sozialen glauben, um zu prüfen, ob bestimmte Methoden zur Aufdeckung kausaler Gesetzmäßigkeiten geeignet sind, ebensowenig wie man an Parapsychologie oder Hexerei glauben muB, um den Erfolg der Anwendung entsprechender Verfahren zu testen. Selbst wenn die Annahme, es gäbe Kausalität in der Soziologie, in keinem einzigen Fall erfüllt wäre, würde dies unserer Kritik nicht die Spitze nehmen. Sie liefe dann nur auf die (vielleicht nicht $\mathrm{zu}$ interessante) These hinaus, daß die diskutierten Methoden ihr Ziel selbst dann nicht erreichen könnten, wenn es vorhanden wäre.

Auch der zweite Einwand mißversteht unsere Intention, denn wir empfehlen Soziologen nicht, auf experimentelle oder quasiexperimentelle Methoden auszuweichen. Dies ist nicht nur aus ethischen Gründen unmöglich, vielleicht würde es auch in dieser Disziplin zu keinen Einsichten führen, weil Experimente aus anderen Gründen inadäquat oder unmöglich sind (auch dafür gibt es in den Naturwissenschaften hinreichend viele Beispiele). Möglicherweise gibt es auch hocheffiziente (aber bisher noch nicht entwickelte) Methoden, die uns die bislang verwehrten Einsichten in die kausalen Mechanismen der sozialen Realität gestatten. Wenn es sie gibt, werden sie keine Ähnlichkeit mit den bislang verwendeten Methoden der Kausalanalyse haben, denn diese erscheinen hochgradig ineffektiv, ja mehr, eher als eine Art Pseudowissenschaft. Allein diese - durchaus starke und folgenreiche These ist zu begründen. Weitere SchluBfolgerungen über die Struktur so- 
zialer Wirklichkeit und adäquate Methoden zu ihrer Erforschung haben wir nicht anzubieten. Skepsis über die Chancen einer derartigen Forschungsstrategie werden allenthalben deutlich werden, aber wir werden nicht versuchen, sie zu grundsätzlichen Thesen über die Struktur sozialer Realität und angemessener Methoden ihrer Erforschung zu verdichten.

\section{Die Strategie der Kritik}

Bislang sprechen wir immer von "Methoden zur kausalen Analyse", "nichtexperimentellen Verfahren" etc. und unsere Kritik zielt auch auf alle Verfahren dieser Art. Um die Diskussion einzugrenzen und möglichst konkret führen zu können, werden wir nur die kausale Analyse nominalskalierter Daten behandeln, wie sie in der Soziologie üblich ist und mit Verfahren wie der Partialtabellenanalyse, ECTA, NONMET etc. betrieben wird. Als typischen Anwendungsfall legen wir dabei eine (nicht mit Zahlen dokumentierte und wirklich durchgefuhrte) Untersuchung zu der Frage "Was sind die Ursachen dafür, daß jemand der Friedensbewegung angehört?" zugrunde. In dieser fiktiven Untersuchung gibt es zwei Gruppen von Individuen. In der einen Gruppe fassen wir alle die Personen zusammen, die der Friedensbewegung angehören oder zumindest mit ihr sympathisieren. Die zweite Gruppe ist das Komplement der ersten Gruppe. Die Frage ist nun, welche Ursachen dafür maßgeblich sind, daß jemand einer der beiden Gruppen (und nicht der anderen) zuzurechnen ist. Möglich sind Ursachen wie Geschlecht, Alter, Bildung, Parteizugehörigkeit, Kriegserfahrung, Aggressivität etc. Von allen denkbaren unabhängigen Variablen unterstellen wir wieder, sie seien dichotomisiert und nominalskaliert. Für den Meßprozeß gehen wir von idealisierenden Annahmen aus:

I) Jede als relevant erscheinende Variable ist fehlerfrei meßbar.

2) Es dürfen beliebig viele unabhängige Variable in die Untersuchung einbezogen werden.

3) Die Zahl der untersuchten Individuen kann beliebig groß gewählt werden.

Alle drei Voraussetzungen sind in wirklichen Untersuchungen nicht zu erfüllen. Die fehlerfreie Meßbarkeit nicht, weil man u.a. erhebliche Probleme hat, den vorkommenden theoretischen Variablen die richtigen Indikatoren zuzuordnen. Die beiden nächsten Voraussetzungen hängen eng zusammen. Denn mit jeder neu eingeführten unabhängigen Variablen verdoppelt sich die Zahl der Zellen des Datensatzes und man ist selbst bei großen Untersuchungen nicht in der Lage, in einer Analyse z.B. Is Variablen zu verarbeiten, weil dann die meisten Zellen zu schwach besetzt sind. Dies kann man nur durch eine Vergrößerung der Zahl der Untersuchten verhindern und mit der dritten Annahme soll dies in beliebigem Umfang möglich sein. 
Warum machen wir diese Voraussetzungen, obwohl sie vollkommen unrealistisch sind? Die Antwort sollte klar sein, denn es geht zunächst darum, die Modelle (bzw. ihre Anwendung) möglichst stark zu machen, weil die dann immer noch verfügbaren Argumente für die angekündigte Kritik ausreichen. Daß eine adäquate Kausalanalyse schon wegen der in ( 1 ) - (3) implizit angesprochenen Probleme scheitern kann, braucht uns daher nicht zu interessieren.

Die übliche Vorgehensweise ist nun: Man wählt ein bestimmtes Analysemodell aus, z.B. ECTA, und spezifiziert ein Kausalmodell, in dem die erwarteten Wirkungen der unabhängigen Variablen auf die abhängigen beschrieben werden. Mit dem Analysemodell wird dann bestimnit, ob die Daten zu dem Modell passen.

Ich habe die Begriffe "Analysemodell", "Kausalmodell". und "Wirkung von Variablen" verwendet. Da diese Begriffe noch häufiger vorkommen und eine andere semantische Belegung als die von mir intendierte zu erheblichen Konfusionen führen kann, sei hier ausdrücklich auf den unterstellten Sinn verwiesen :

- "Analy severfahren", "Kausalanalyseverfahren" werden synonym verwendet. Wo Mißverständnisse auszuschließen sind, wird auch "Kausalanalysemodell" gebraucht.

- Unter "Analysemcdell" wird ein bestimmtes statistisches Analyseverfahren wie ECTA, NONMET verstanden, nicht aber eine konkrete Anwendung in einer bestimmten Untersuchung.

- Unter einem "Kausalmodell" verstehe ich die Beschreibung der angenommen kausalen Zusammenhänge zwischen der unabhängigen und der abhängigen Variablen. Ein Kausalmodell enthält demrach Beschreibungen wie "es besteht ein Zusammenhang zwischen Alter und der Teilnahme an der Friedensbewegung" etc. Damit ein bestimmtes Kausalmodell mit einem Analysemodell gerechnet werden kann, müssen solche Beschreibungen in die Sprache des Analysemodells übersetzt werden.

- "Wirkung einer Variablen" ist eine unsinnige, aber in dem soziologischen Jargon geradezu eingebaute Verwendungsweise, so da $B$ wir nicht darauf verzichten wollen. Da der Mißbrauch stets mit demonstrativer Lässigkeit erfolgt, muB man wohl eine solide Kenntnis der korrekten Verwendung unterstellen können, so daß hier auf eine Begriffsexplikation verzichtet werden kann. Im übrigen werden wir weiter unten ausführlich auf wichtige Aspekte der Frage "wie wirkt die Kausalität?" eingehen, womit die Mißverständnisse auf das in Wissenschaftskreisen unverzichtbare AusmaB reduziert werden sollten.

Wir haben weiter oben auf Analyseverfahren wie ECTA und NONMET verwiesen, deren Anwendung (auch) das Ziel unserer Kritik ist. Der Leser 
- insbesondere der in der Verwendung solcher Analysepakete geschulte Leser - erwartet deswegen vielleicht eine detailbezogene Kritik an einzelnen statistischen Merkmalen der Verfahren und daher praxisgerechte Ratschläge für deren Verwendung. Beides werden (und können) wir nicht vortragen. Es geht in dem vorliegenden Aufsatz nicht um die Detailkritik einzelner Analyseverfahren mit dem Ziel einer Verbesserung, sondern um eine fundamentale Kritik, die den Nutzen der Anwendung solcher Verfahren generell bezweifelt. Damit soll nicht die Möglichkeit einer Verbesserung im Detail bestritten werden und ebensowenig die Möglichkeit sinnvoller Anwendungen in speziellen (aber für die Kausalanalyse) atypischen Situationen. Für Beispiele wie das genannte und auch für die in Lehrbüchern referierten Anwendungen (von denen man eigentlich unterstellen sollte, da B sie die Vorzüge der Verfahren besonders gut herausarbeiten) erscheinen die Verfahren gleichwohl ungeeignet.

Nun ist dies eine drastische und - so sie richtig sein sollte -weitreichende Kritik, deren Ankündigung Zweifel wecken muB. Denn wer so steile Thesen aufstellt, operiert oft mit philosophisch einschlägigen, aber praktisch folgenlosen Argumenten. Er mag dann zwar 'im Prinzip' unwiderlegbar sein (wie z.B. ein Solipsist), nur hat man das deutliche Gefühl, einer philosophischen Spinnerei aufgesessen zu sein, die man schnellstens wieder vergißt. Dieser Verdacht mag hier besonders leicht entstehen, weil Kausalität ein philosophisches Standardthema ist, dessen Diskussion uber mehrere Jahrhunderte zu einer Fülle von Einsichten geführt hat, die für den mit kausalen Gesetzen arbeitenden Wissenschaftler irrelevant sind.

Es ist deswegen außerordentlich wichtig, vor jeder Detailargumentation zu klären, warum wir diese "philosophische" Strategie nicht einschlagen werden. Dazu mu $B$ verdeutlicht werden, wie man so weitreichende Thesen, wie die von uns vertretenen, begründen kann, ohne dabei auf zwei Typen von Argumenten zurückzugreifen. Wir haben nämlich weder

(I) Argumente über die tatsăchliche Struktur des Gegenstandsbereiches, noch wollen wir

(2) Argumente verwenden, die sich auf technische Details einzelner Verfahren beziehen.

(I) scheidet aus, weil dieses Wissen nicht zur Verfügung steht und mit (2) müBte man immer den Einwand gewärtigen, ein nicht diskutiertes Verfahren oder die nicht berücksichtigte Modifikation eines bekannten Verfahrens würden von der vorgetragenen Kritik nicht berührt.

Aber fingieren wir für einen Augenblick, wir hätten das in ( $\mathrm{r}$ ) beschriebene Wissen und könnten eine vollständige Beschreibung jedes Bereichs der sozialen Wirklichkeit durch kausale Gesetze angeben. Für unser Beispiel wäre es dann möglich, alle Variablen (und die Art ihrer Wirkung) zu be- 
nennen, die für die Sympathie bzw. Nicht-Sympathie bezüglich der Friedensbewegung verantwortlich sind. Im Fall eines deterministischen Systems lieBe sich dann das in Frage kommende Verhalten mit Sicherheit voraussagen, sofern wir in der Lage sind, die Ausgangsbedingungen zu messen, was ebenfalls unterstellt werden soll.

Dann wäre der Beweis unserer These sehr einfach: Wir würden zeigen, warum es keine Möglichkeit gibt, die tatsächlichen kausalen Verhältnisse mit den Analysemodellen aufzudecken. Wie man einen solchen Beweis führen könnte, braucht hier im Detail nicht zu interessieren, solange man die Strategie akzeptiert, um ein solches 'no-go-Theorem' zu begründen: Man würde einfach typische Anwendungsfälle für kausale Analysen betrachten und sich überlegen, warum die tatsächlichen Verhältnisse für die verwendeten Analysemodelle viel zu kompliziert sind. In dem Satz "man kann unter diesen Bedingungen die wahre Struktur der kausalen Verhältnisse nicht finden" hat das "kann" freilich eine andere Bedeutung wie in "man kann keinen Kreis quadrieren" oder "man kann kein perpetuum mobile bauen". Es hat eher die Verwendung wie in der Aussage "ein Laie kann unmöglich das Sicherheitssystem eines Bankcomputers knacken, auch wenn es prinzipiell kombinatorisch möglich ist". "Ist nicht möglich","kann nicht" besagt demnach soviel wie "die Aufgabe ist viel zu kompliziert, als daB sie gelöst werden könnte, obwohl es rein kombinatorisch möglich ist". Eine Lösung wäre ein Zufallstreffer.

Leider kennen wir die kausalen Gesetze der sozialen Realität nicht und wenn wir sie kennen würden, dann wäre die vorzutragende Kritik überflüssig, weil die kritisierten Analysemodelle ohnehin von niemandem verwendet würden. Wir müssen demnach anders vorgehen. Wenn jemand nach kausalen Gesetzen in der Soziologie sucht, dann macht er bestimmte, sehr allgemeine Annahmen. Diese Annahmen ergeben sich zwingend aus der Voraussetzung, die soziale Realität sei nach kausalen Gesetzmäßigkeiten strukturiert. Eine kausale Modellwelt nennen wir ein Modell, das mit diesen Annahmen verträglich ist. In grober Näherung kann man ein Modell als eine Vorstellung darüber auffassen, wie der jetzt beobachtete Weltzustand (die multivariate Tabelle) durch kausale Mechanismen zustande gekommen sein könnte. Natürlich gibt es eine Unzahl möglicher Weltverläufe, die zu dem beobachteten Zustand geführt haben könnten. Aufgabe eines Kausalanalyseverfahrens ist es darum, für ein bestimmtes Kausalmodell zu prüfen, ob es zu dem beobachteten Weltzustand paBt. Die Strategie unserer Kritik besteht nun darin, zu zeigen, daß wir i.a. nicht in der Lage sind, ein bestimmtes Modell auszuzeichnen, weil die Zahl der Parameter, mit denen wir spielen können, viel zu groß ist. Es geht im folgenden also darum, die Annahmen bzw. Parameter herauszuarbeiten, die wir kennen müBten, um Kausalanalyse betreiben zu können. 
Bevor dieser Gedanke etwas weiter ausgeführt wird, soll der Begriff "kausale Modellwelt" erläutert werden. Wir betrachten dafür wieder unser Beispiel. Wenn die Differenz "friedensbewegt - nicht friedensbewegt" durch den kausalen Einflu B der unabhängigen Variablen zustande kommt, dann haben wir grundsătzlich folgenden Ablauf:

(I) Zustand der Welt zur Zeit $\mathrm{T}_{\text {I }}$

(2) Wirken der kausalen Mechanismen

(3) Zustand der Welt zur Zeit $\mathrm{T}_{2}$

Eine kausale Modellwelt ist eine genaue Beschreibung dieser drei Größen. "Modell", weil wir wesentliche Abstraktionen von der realen Welt vornehmen: Wir betrachten z.B. nur dichotome Variable, lassen nur bestimmte Wirkungen zu (keine Wirkungen der unabhängigen Variablen aufeinander), etc.

Je nachdem wie man den Zustand der Welt zu $T_{I}$ einschätzt und was man als kausale Gesetze annimmt, wird man zu ganz unterschiedlichen Werten bei $T_{2}$ kommen. Aber damit sind wir schon wieder bei irrealen Annahmen. Wir kennen weder den Zustand der Welt zur Zeit $T_{I}$, noch die kausalen Gesetze, sondern nur den $\operatorname{im}(n+r)$-dimensionalen ( $n$ unabhängige Variable) Datenkubus eingefrorenen Zustand der Welt zum Zeitpunkt $\mathrm{T}_{2}$. Aber wir dürfen unserer Phantasie freien Lauf lassen, wie die Welt zu $T_{I}$ gewesen sein könnte und wir dürfen auch unserer Phantasie bezüglich der möglichen kausalen Gesetze freien Lauf lassen und so klären, welchen Zustand wir bei $\mathrm{T}_{2}$ erhalten hätten. Welche Grenzen wir unserer Phantasie dabei auferlegen müssen, i.e. welche kausalen Modellwelten zugelassen werden dürfen, braucht uns jetzt nicht zu interessieren, denn damit werden wir uns später ausführlich auseinanderzusetzen haben. Der Dreh in der Argumentation ergibt sich nun aus der Frage, ob die Klasse der kausalen Modellwelten so eingeschränkt werden kann, daß es nur einen einzigen Verlauf gibt, der zu dem Zustand $T_{2}$ führt und alle anderen Verläufe, die 'mehr oder weniger dicht' an den 2 ustand $\mathrm{T}_{2}$ heranführen, sich von der ersten kausalen Modellwelt entsprechend wenig unterscheiden. Schematisch läßt sich das Ausgangsproblem so charakterisieren:

In einer kausalen Modellwelt gilt: wir kennen ( $I$ ) und (2)

und können dann eindeutig

auf (3) schließen. Oder, griffiger formuliert,

(1) und (2) $\Rightarrow(3)$

In der Analyse ist das Problem umgekehrt
(3)
(I) und (2).

Man konstruiert kausale Modellwelten und schaut, ob man hinreichend dicht an den Weltzustand zum Zeitpunkt $\mathrm{T}_{2}$ herankommt. Unser Problem wira 
nie darin bestehen, kein kausales Modell zu finden, mit dem wir dicht genug an $\mathrm{T}_{2}$ herankommen, sondern es wird gerade umgekehrt darin bestehen, die meisten Verläufe durch zusätzliche Annahmen auszuschließen.

Die für unsere Kritik entscheidende Frage ist, ob die Klasse der möglichen Modellwelten mit gut begründeten Annahmen hinreichend eingegrenzt werden kann. Oberflächlich betrachtet mag die Antwort positiv ausfallen, weil man sich stets auf einschlägige Theorien beziehen könnte. Aber eine nähere Analyse zeigt, wie problematisch das Argument ist. Denn für die Annahme, die wir machen müßten, um die Klasse der kausalen Modellwelten hinreichend einzuschränken, haben wir i.a. keine Theorien zur Verfügung. V/as bleibt ist eine petitio principii: Man trifft die Annahmen so, daß genau das bei der Analyse herauskommt, was man haben möchte. Dabei sollte man sich vor Augen halten, daß unsere Kritik in zwei Richtungen zielt:

- durch die Explikation notwendiger aber unerfüllbarer Annahmen soll gezeigt werden, warum soziologische Kausalanalyse die beanspruchten Erkenntnisziele nicht einlösen kann;

- darüberhinaus haben wir auch - indirekt - konkrete Analysemodelle mit im Visier, weil sie nicht geeignet sind, für die Analyse wesentliche Parameter zu berücksichtigen.

Unsere Antwort auf die entscheidende Frage nach einer Eingrenzung der Klasse möglicher Modellwelten ist eindeutig negativ und kann nur dann 'positiv' werden, wenn die Klasse der kausalen Modellwelten auf eine nicht zu rechtfertigende Weise durch a priori Annahmen eingeengt würde. Selbstverständlich kann man Eindeutigkeit durch die Einführung starker Randbedingungen erzwingen, aber dann setzt man das zu Beweisende voraus. In diesem Falle macht man (nicht begründbare) Annahmen über die Wirkung bestimmter Kausalfaktoren, um dann zu schließen, daß nur diese Kausalfaktoren wirksam gewesen sind.

Um diese These ausführen zu können, müssen wir die kausale Modellwelt näher charakterisieren. Dazu brauchen wir

- eine Beschreibung des Ausgangszustandes (das, was vorher mit (I) bezeichnet wurde);

- eine genaue Beschreibung der wirksamen kausalen Kräfte und die Art ihrer Wirkung (das, was vorher mit (2) bezeichnet wurde und zusammen mit (I) zu (3) führt).

Für die Konstruktion der kausalen Modellwelt müssen einige Vereinfachungen vorgenommen werden: Die Individuen können nur in dichotomen $\mathrm{Zu}$ standsbeschreibungen charakterisiert werden. (Wenn wir vom "Weltzustand zum Zeitpunkt $\mathrm{T}_{\mathrm{I}}$ " reden, dann meinen wir die Verteilung der Individuen 
in dem Datenkubus zu dem Zeitpunkt $T_{r}$. Dabei werden die auf die Individuen wirkenden kausalen Kräfte nicht berücksichtigt. Deswegen wäre es genauer, von einem "nicht-kausalen Weltzustand zum Zeitpunkt $\mathrm{T}_{\mathrm{I}}$ " zu reden. Die kausalen Weltzustände werden wir weiter unten charakterisieren.) Alle möglichen feineren Informationen werden durch die Dichotomisierung vernachlässigt. Es gibt $n$ unabhängige und eine abhängige Variable, wobei die $n$ unabhängigen Variablen wechselseitig unabhängig sein sollen. Weiterhin wird eine Art "statische Kausalität" unterstellt. Zu dem Zeitpunkt $T_{\text {}}$ haben wir einen nicht-kausalen Weltzustand. Dann fügen wir - je nach dem spezifischen Kausalmodell - die kausalen Kräfte hinzu. Die kausalen Kräfte können nur Veränderungen in der Verteilung der abhängigen Variablen bewirken (oder einer solchen Veränderung entgegenwirken). Die Art der Verănderung läßt sich berechnen und in dem Datenkubus als Verschiebung durchführen.

Der Weltzustand im Zeitpunkt $\mathrm{T}_{\mathrm{I}}$ geht in einen Weltzustand zum Zeitpunkt $T_{2}$ uber. Solange keine neuen kausalen Kräfte hinzutreten, verändert sich der Weltzustand zu dem Zeitpunkt $T_{2}$ nicht mehr. Das ist es, was mit "statischer Kausalität" gemeint ist: Die Wirkung der kausalen Kräfte entfaltet sich "auf einen Schlag", und es wird nicht eine Veränderung in Gang gesetzt, die zu einem dynamischen Gleichgewicht führt. Auf dieser Grundlage lassen sich weitere wichtige Begriffe für die Charakterisierung der kausalen Modellwelt einführen. Zu unterscheiden ist zwischen

- $\quad$ einem kausalen Weltzustand mit Verschiebungsdisposition

und

- einem kausalen Weltzustand ohne Verschiebungsdisposition.

In dem ersten Fall führen die angenommenen kausalen Kräfte (wenn man ihre Wirkung berechnet) zu einer Veränderung in der Verteilung der abhängigen Variablen, in dem zweiten Fall führen die kausalen Kräfte nicht zu einer Veränderung. Diese Unterscheidung ist deswegen wichtig, weil sich die kausalen Kräfte so ausbalancieren können, daß eine Veränderung in der Verteilung der abhängigen Variablen nicht zustande kommt. Wenn wir beispielsweise von dem Weltzustand $T_{1}$ in den Weltzustand $T_{2}$ übergegangen sind, dann wird es ohne neue kausale Kräfte keine weiteren Veränderungen in der Verteilung der abhăngigen Variablen geben. Aber deswegen darf der Weltzustand zum Zeitpunkt $T_{2}$ nicht als nicht-kausal beschrieben werden, denn es können z.B. kausale Kräfte vorhanden sein, die einer Veränderung entgegenwirken. Wir unterscheiden demnach zwischen Weltzuständen, in denen kausale Kräfte wirken und solchen, in denen kausalen Kräfte wirken. Es ist klar, daß im zweiten Fall auch keine Verschiebung von Individuen zustande kommen kann. Aber im ersten Fall muß es nicht zu einer Verschiebung kommen, weil sich die kausalen Kräfte kompensieren können. Dann liegt keine Verschiebungsdisposition vor. 
Die kausale Modellwelt ist damit so weit charakterisiert wie das möglich ist, ohne im einzelnen auf die kausalen Gesetze einzugehen, die zu den Veränderungen in der Modellwelt führen. Die oben eingeführten Begriffe werden verständlicher, wenn sie vor dem. Hintergrund der kausalen Mechanismen verstanden werden, die wir jetzt einführen. Dieser Teil der Arbeit ist insofern nicht ganz unproblematisch als wir die vielen einschlägigen philosophischen Beiträge zu diesem Thema allenfalls nur streifen, da sie nicht unter dem für uns erkenntnisleitenden Gesichtspunkt subsumiert sind. Die Modellvorstellungen sind bewuBt einfach gehalten, weil wir hier wie in anderen Passagen der Arbeit nur das absolut notwendige Ausma B an Verkomplizierung in Kauf nehmen wollen, um nicht das Gegenargument zu provozieren, die Welt sei viel einfacher als von uns beschrieben und die Kritik an den Kausalanalyseverfahren daher unberechtigt. Ganz im Gegenteil gilt die Behauptung: Die Welt ist zumindest so kompliziert wie von uns beschrieben und die gängigen Verfahren werden noch nicht einmal damit fertig.

\section{Die Wirkung der kausalen Kräfte}

Alles was wir durch die Wirkung der kausalen Kräfte in der kausalen Modellwelt verändern können, ist die Verteilung der abhängigen Variablen. Konkret heißt dies :

- Individuen sind $\mathrm{zu} \mathrm{T}_{\mathrm{I}}$ "friedensbewegt" und $\mathrm{zu} \mathrm{T}_{2}$ "nicht friedensbewegt".

- Individuen sind $\mathrm{zu} \mathrm{T}_{\mathrm{I}}$ "nicht friedensbewegt" und $\mathrm{zu} \mathrm{T}_{2}$ "frieden sbewegt".

Wenn beispielsweise "Angst vor einem Krieg" eine Ursache für den Übergang von nicht friedensbewegt zu friedensbewegt ist, dann kann die Wirkung der kausalen Kraft darin bestehen, daß $30 \%$ aller nicht friedensbewegten Individuen friedensbewegt werden. Wir gehen von nicht deterministischen kausalen Kräften aus. Dies ist pragmatisch geboten aber theoretisch nicht ganz unproblematisch, denn schließlich könnten sich die nicht deterministischen kausalen Kräfte bei einer feineren Analyse als deterministisch herausstellen. In den folgenden Ausführungen werden Gründe angeführt, warum dies so sein könnte, obwohl die Frage explizit nicht erörtert wird.

Neben den verschiebenden kausalen Kräften gibt es die festhaltenden kausalen Kräfte. Beispielsweise kann jemand Angst vor einem Krieg haben und friedensbewegt sein, wobei unterstellt wird, Angst vor einem Krieg sei nicht die einzige Ursache, sondern es komme die Kritik an den hohen Rüstungsausgaben als Ursache hinzu. Jede der beiden kausalen Kräfte wäre für sich allein ausreichend, um aus einem nicht Friedensbewegten einen Friedensbewegten zu machen. Aber trotzdem kann eine der beiden verschiebenden Kräfte nicht einfach mit dem Argument weggelassen werden, 
die andere sei für die Verschiebung ausreichend. Das wird sofort deutlich, wenn man eine in die andere Richtung wirkende Kraft betrachtet, etwa "Angst vor der Sowjetunion". Angenommen ohne jede andere kausale Kraft führe Angst vor der Sowjetunion zu einer Verschiebung von friedensbewegt zu nicht friedensbewegt. Wirken aber die beiden anderen Kräfte auf das entsprechende Individuum ein, dann sind sie geeignet, die verschiebende Kraft zu kompensieren, so da $B$ die betreffende Person friedensbewegt bleibt, obwohl sie ohne jede Gegenkraft in die Kategorie nicht friedensbewegt verschoben würde. Genau deshalb ist es nicht gleichgültig, ob auf ein Individuum eine oder mehrere verschiebende Kräfte wirken, weil die verändernden Kräfte nach der Verschiebung weiterwirken und so zu festhaltenden Kräften werden. Wer aus Angst vor einem Krieg und wegen der Kritik an den hohen Rüstungsausgaben zu der Friedensbewegung stößt, der wird von diesen beiden Motiven dort stärker festgehalten als jemand, der "nur" wegen der Kritik an den hohen Rüstungsausgaben friedensbewegt ist.

Mit diesen Vorüberlegungen kann die kausale Modellwelt näher beschrieben werden: Angenommen wir haben insgesamt $k$ Individuen $I_{1}, I_{2}, \ldots$, $I_{k}$, verteilt auf die beiden Zellen "friedensbewegt" und "nicht friedensbewegt". Für jedes dieser k Individuen gilt nun

(i) entweder wirkt keine kausale Kraft auf das Individuum ein,

(ii) oder es wirken $m_{i}$ festhaltende und $n_{i}$ verschiebende Kräfte auf $\mathrm{I}_{\mathrm{i}}, \mathrm{m}_{\mathrm{i}}+\mathrm{n}_{\mathrm{i}} \geq \mathrm{I}$.

Tritt der Fall (i) ein oder ist $m_{i} \geq n_{i}$ dann bleibt das Individuum in seiner Zelle, weil die verschiebenden Kräfte zu schwach sind, um die festhaltenden Kräfte zu kompensieren. Andernfalls, i.e. $n_{i}>m_{i}$, wird das Individuum von seiner Zelle in die andere verschoben. In seiner neuen Zelle wird es dann mit der verschiebenden Kraft festgehalten.

Damit ist der fundamentale Verschiebungsmechanismus in der kausalen Modellwelt beschrieben. Man berechnet einfach die Bilanz der kausalen Kräfte auf ein Individuum und verändert entsprechend seinen Status.

Mit diesen Erläuterungen wird auch der Unterschied zwischen einer kausalen Modellwelt mit und einer kausalen Modelwelt ohne Verschiebungsdisposition deutlich. In dem ersten Fall ergibt sich aus der Summe der Kräfte, da $B$ bei der Wirkung der kausalen Kraft das Individuum die Zelle wechselt, in dem zweiten Fall, daß es bei dem Wirken der Kräfte in der Zelle bleibt oder in der Zelle festgehalten wird. Beispiel: Ein Individuum ist in der Zelle "nicht friedensbewegt". Es ist pro CDU (Wirkung "friedensbewegt nicht friedensbewegt") und hat Angst vor einem Krieg (Wirkung "nicht friedensbewegt - friedensbewegt"). Wenn der Betreffende nun nicht friedensbewegt ist und keine weiteren kausalen Kräfte auf ihn einwirken, dann wird er nicht friedensbewegt bleiben. Denn die beiden kausalen Kräfte 
kompensieren sich und demnach gibt es keine Verschiebungsdisposition. Wenn der Betreffende weiterhin den Wehrdienst verweigert und es gilt die Wirkung ("nicht friedensbewegt $\rightarrow$ friedensbewegt") dann liegt eine Verschiebungsdisposition vor. Denn wenn die kausalen Kräfte wirken, dann wird er die Einstellung wechseln und von nicht friedensbewegt zu friedensbewegt übergehen.

Eine graphische Darstellung hilft, einen schnelleren Uberblick zu bekommen. Für jede kausale Kraft, die in Richtung der anderen Zelle - also verschiebend - wirkt, zeichnen wir einen Pfeil in Richtung dieser Zelle. Für jede kausale $\mathrm{Kraft}$, die ein Individuum in der Zelle festhält, in der es sich befindet, zeichnen wir einen Pfeil, der in die Zelle hineinweist. Für das obige Beispiel sieht das so aus:

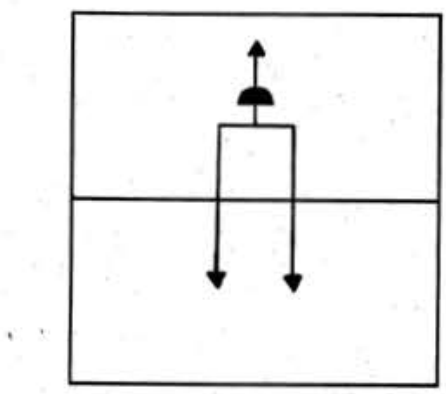

\author{
nicht \\ friedensbewegt \\ friedensbewegt
}

Der nach oben weisende Pfeil steht für "pro CDU"; die nach unten weisenden Pfeile für "Angst vor einem Krieg" und "verweigert den Wehrdienst". Würden die kausalen Kräfte wirken, dann săhe das Bild so aus:

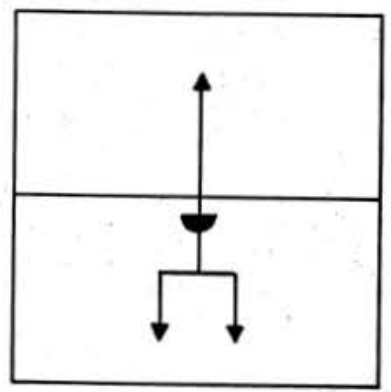

nicht

friedensbewegt

friedensbewegt 
Das Beispiel macht einen wichtigen Punkt deutlich. Ob ein Individuum eine Verschiebungsdisposition hat, hängt von der Zelle $a b$, in der es sich befindet. Das ist inhaltlich einleuchtend, denn wenn jemand bereits friedensbewegt ist (aus Gründen oder kausalen Krăften heraus, die in der Analyse nicht berücksichtigt werden) und es wirken mehr kausale Kräfte in Richtung friedensbewegt als umgekehrt, dann wird sich nichts verăndern, während im anderen Falle sich seine Einstellung verändern wird.

Wenn man der obigen Ausführung kritisch folgt, dann stößt man auf ein Problem, das hier zumindest erwähnt werden soll, weil es für jede Form soziologischer Kausalanalyse erheblich ist. Es war die Rede von Individuen, die in einem der beiden Zustände der abhängigen Variablen sind. Das war notwendig, weil jede Kausalanalyse von dem Zustand ausgehen muB, in dem die untersuchten kausalen $\mathrm{Kräfte}$ nicht wirken. Aber andererseits muB man fragen, wie die Personen in diesen Zustand hineingeraten, denn die Unterstellung, die seien "einfach so drin", ist zu simpel. Die Annahme, es seien uns unbekannte kausale Kräfte, die die Individuen in diesen Zustand gebracht haben und dort halten, ist naheliegend, zumal es auf die Frage "wie sollen sie denn sonst in den Zustand geraten sein?" nur schwer eine Antwort gibt. Solange man darauf keine überzeugende Antwort finden kann - und Pfeile, die auf unbekannte Faktoren verweisen, sind sicher keine - ist gegen jede Analyse ein schwer widerlegbarer Einwand vorzubringen: da B nämlich die Berücksichtigung aller relevanten Faktoren, oder relevanter kausaler Kräfte - ein ganz anderes Bild ergäbe. Wir möchten diesen Einwand jetzt nicht diskutieren, weil wir später in einem anderen Kontext darauf zurückkommen werden, aber wir halten es für sinnvoll, dieses wichtige Problem wenigstens zu erwähnen. Vorerst wird deswegen weiter von Individuen gesprochen, die in bestimmten Ausgangszuständen sind, nämlich friedensbewegt/nicht friedensbewegt, ohne $\mathrm{da} B$ untersucht wird, wie die Individuen in diese Zustände kommen.

Ersichtlich wird die Dynamik der Veränderung in der kausalen Modellwelt nicht berücksichtigt. Die Verschiebung erfolgt "auf einen Schlag" und nicht so, wie man sich realistischerweise vorstellen muB, als Einpegeln eines dynamischen Gleichgewichtszustandes. Die Rechtfertigung für diese Vereinfachung liegt auf der Hand: Für die Bestimmung der Stärke einzelner Effekte ist es uninteressant, wie die Veränderung zeitlich abläuft. Würde man den zeitlichen Verlauf der Veränderung berücksichtigen, dann würde jedes der hier behandelten Modelle ("Veränderung auf einen Schlag") in eine Reihe von Untermodellen aufgespalten, was die Aufgabe für Kausalanalyseverfahren nochmals verkomplizierte: Dann müBte zusătzlich die Dynamik der Veränderung erschlossen werden.

Noch ist allerdings das Wirken der kausalen Kräfte nicht vollständig charakterisiert: Bislang wurden alle kausalen Kräfte als gleich stark betrachtet, was sich in folgender Additionsregel ausdrückt: Wenn k Kräfte aus 
der Zelle herauswirken und $\mathrm{n}$ in sie hinein, dann wirkt auf das Individuum, d.u.n.d. eine verschiebende Kraft aus der Zelle heraus, wenn $k>n$ gilt, d.h. es muB - sehr anschaulich gesprochen - mehr kausale Kräfte geben, die ein Individuum aus einem Zustand herausziehen als es Krăfte gibt, die es in diesem Zustand festhalten.

\section{Notwendige Voraussetzungen für kausale Analysen I: \\ Die Stärke kausaler Kräfte}

Die Annahme jeweils gleich starker kausaler Kräfte ist aber nicht generell gültig und allenfalls als technische Vereinfachung für die Konstruktion von Schätzverfahren motivierbar. Beispielsweise kann jemand der Friedensbewegung angehören, weil er selbst die Grausamkeit eines Krieges erfahren hat und deswegen jede Art von Krieg kategorisch ablehnt (rte kausale Kraft) und trotzdem den Habitus der Repräsentanten dieser Bewegung mißbilligen, weil er ansonsten sehr konservative Wertvorstellungen hat (2te kausale $\mathrm{Kraft})$. Des weiteren mögen ihn an der Friedensbewegung Formen des zivilen Ungehorsams stören (3te kausale Kraft). Wir müßten nun schließen: Es gibt 2 von der Friedensbewegung wegziehende Kräfte und eine festhaltende, also kann der Betreffende nicht friedensbewegt sein. Denn entweder ist er es ohnehin nicht, oder die kausale Kraft verschiebt ihn in die Kategorie "nicht friedensbewegt". Das aber ist inhaltlich nicht plausibel, denn ein Kriegsgegner aus den genannten Gründen wird auf Seiten der Friedensbewegung stehen, auch wenn es schwächere Gründe gibt, die ihn von dieser Bewegung wegbringen. Diese Art zu schließen entspricht dem Alltagsverständnis, wo es schlicht absurd wăre, wenn z.B. vor einer Entscheidung die Zahl der Gründe für eine Handlung und die Zahl der Gründe gegen eine Handlung uns durch eine einfache Substraktion festlegen würden, was man tun soll. Wir unterscheiden vielmehr im Alltag zwischen starken und schwachen Motiven, mehr oder weniger guten Gründen, und müssen auch in einer kausalen Analyse, in der Gründe und Motive als kausale Kräfte interpretiert werden, an dieser Unterscheidung festhalten.

Das Beharren auf der gleichen Stärke aller kausalen Kräfte wäre weltfremd und würde die Modellierung kausaler sozialer Beziehungen nicht gestatten. Man könnte geradezu umgekehrt sagen, $\mathrm{da} B$ es häufig einen entscheidenden Grund für eine Handlung oder Einstellung gibt und daB dieser Grund alle anderen entgegenstehenden leicht kompensiert und so eine Veränderung verhindert oder bewirkt, je nachdem, wie der vorherige Zustand gewesen ist. Man denke etwa an Wahlentscheidungen, bei denen Arbeitslosigkeit, Umweltkrisen etc. für viele das ausschlaggebende Motiv sind, sich für eine Partei zu entscheiden, obwohl sie eine Reihe von Gründen kennen, die gegen ihre Wahlentscheidung sprechen. 
Deswegen ist eine Modifikation unseres bisherigen Modells erforderlich: Jeder kausalen Kraft wird eine Stärke zugeordnet, so daß sich die Gesamtwirkung der kausalen Kräfte aus ihrer Stärke und Zahl ergibt. Die vorherigen Überlegungen stellen demnach einen Spezialfall dar, bei dem alle kausalen Kräfte mit der Stärke I gewichtet wurden. Wie sich die Bilanz der kausalen Kräfte durch die Berücksichtigung von Stärken verändern kann, zeigt die Analyse des verwendeten Beispiels. Wir hatten dort drei kausale Krăfte:

pro Friedensbewegung: lehnt jede Art von Krieg kategorisch ab;

contra Friedensbewegung: hat konservative Wertvorstellungen/stört sich an Formen des zivilen Ungehorsams.

Ohne Gewichtung der Stärke gibt es demnach eine resultierende verbleibende Kraft zu "nicht friedensbewegt". D.h. der Betreffende ist entweder nicht friedensbewegt, oder er wird es durch die Wirkung der drei kausalen Kräfte. Um dieses kontraintuitive Resultat zu verhindern, werden Stärkefaktoren eingefüht:

"Lehnt jede Art von Krieg kategorisch ab"

"Hat konservative Wertvorstellungen"

"Stört sich an Formen zivilen Ungehorsams"

Dies ergibt eine Bilanz der kausalen Kräfte von $5-(2+1)$, d.h. der $\mathrm{Be}-$ treffende ist entweder friedensbewegt, oder er wird es durch die Wirkung der drei kausalen Kräfte.

An dieser Stelle wird man zwei kritische Fragen formulieren müssen:

- warum wurden gerade Stärkefaktoren 5, 2 und I gewăhlt und nicht etwa 8 , 5 und 2 ?

- warum werden die Stärkefaktoren für die kausalen Kräfte und nicht für die Wirkung der kausalen Kräfte auf einzelne Individuen angesetzt?

Auf die erste Frage kann es nur eine sehr vorsichtige Antwort geben, in der nochmals die Notwendigkeit der Berücksichtigung von Stärkefaktoren hervorgehoben wird. Offensichtlich gibt es kausale Ursachen von unterschiedlicher Bedeutung, so daß nicht an der Notwendigkeit der Modellierung unterschiedlicher Stärken, sondern nur an speziellen Modellvorstellungen gezweifelt werden kann. Beispielsweise könnte man eine qualitative Bewertung vornehmen, indem man z.B. Festsetzungen der folgenden Art trifft:

(i) "lehnt jede Art von Krieg kategorisch ab" ist eine stärkere kausale $\mathrm{Kraft}$ als alle anderen kausalen Krăfte zusammen; 
(ii) "hat konservative Wertvorstellungen" ist stärker als "stört sich an Formen des zivilen Ungehorsams";

etc.

Auf diese Weise gibt man nur qualitative Unterschiede zwischen kausalen Kräften an, ohne sich um die numerische Stärke kümmern zu müssen. Das dürfte gerade für soziologische Anwendungen attraktiv sein, nur hat diese Vorgehensweise eine unangenehme Nebenfolge: Die Angabe nur qualitativer Relationen führt zumeist dazu, daß man in vielen Fällen keine Entscheidung über die resultierende Wirkung der kausalen Kräfte treffen kann. Angenommen, wir hätten bezüglich der drei kausalen Kräfte in dem Beispiel nur die folgenden Angaben gemacht:

(i) "lehnt jede Art von Krieg kategorisch ab" hat eine größere kausale Stärke als "hat konservative Wertvorstellungen";

(ii) "hat konservative Wertvorstellungen" hat eine größere kausale Stärke als "stört sich an Formen des zivilen Ungehorsams".

Aus (i) und (ii) kann man nicht schließen, daB "lehnt jede Art von Krieg kategorisch ab" stärker ist als die beiden anderen kausalen Kräfte zusammen. Da man solche Informationslücken vermeiden muB, um in jedem Fall die Resultierende der kausalen Kräfte berechnen zu können, wird man es bei nur ordinalen Angaben mit einer Unzahl von Bedingungen zu tun haben. Will man dies vermeiden, dann bleibt nur der Übergang zu metrischen Annahmen. Damit ist man wieder bei der - polemischen - Ausgangsfrage, woher man denn wisse, daB die kausale Stärke 5 und nicht etwa 8 betrage.

Es wäre vermessen, wenn man versuchte, metrische kausale Stärken zu rechtfertigen. Was wir allenfalls haben, sind plausible Vermutungen über die Rangordnung von Stärken und das auch nur bezogen auf einzelne Individuen, und selbst hier muB man bezweifeln, ob die Rede von Plausibilität auch dann berechtigt ist, wenn man es mit IO-Is unabhängigen Variablen zu tun hat und demnach mit hunderten von Stärkevergleichen zwischen kausalen Effekten.

Aber daraus darf man gerade nicht schluBfolgern, die Berücksichtigung von Stärken sei in einer kausalen Analyse überflüssig: Der Gang der Argumentation muB gerade umgekehrt sein! Weil die kausale Stärke wichtig ist für die Berechnung der resultierenden kausalen Kraft, müssen wir entweder zu plausiblen Annahmen über die kausalen Stärken gelangen, um sie in die Analyse einzubringen - was oft nur sehr begrenzt möglich ist - oder wir müssen die kausale Stärke aus den vorgegebenen Daten erschließen. Wie man sich leicht überlegen kann, ist auch der zweite Weg nicht gangbar. Daraus folgt aber nur ein Argument gegen die praktizierte Form kausaler Analyse in der Soziologie, denn eine kausale Analyse kann nur gelin- 
gen, wenn ein Wissen zur Verfügung steht, das wir nicht haben und mit diesem Verfahren auch nicht erlangen können.

Noch verfahrener ist die Situation, wenn man fragt, wie man "kausale Stärke" prädizieren soll. Soll man sagen, eine kausale Kraft habe eine bestimmte Stärke, oder darf man nur davon reden, eine kausale Kraft habe eine bestimmte Stärke bezüglich einzelner Individuen? Genau genommen scheint mir nur die letzte Redeweise zulässig zu sein, weil eine kausale Kraft wie "lehnt jede Art von Krieg kategorisch ab" auf verschiedene Individuen verschieden stark wirken kann. Jedenfalls gibt es keinen guten Grund für die Annahme, eine kausale Kraft würde auf alle Individuen gleich stark wirken. Es sei denn, man argumentiert, "ceteris paribus" wirke die kausale Kraft gleich stark auf alle Individuen, aber da "lehnt jede Art von Krieg kategorisch ab" nie in reiner Form, sondern nur als Teil einer Interaktion auftritt, wobei bei einzelnen Individuen die interaktiven Effekte unterschiedlich sind, gelangt man so indirekt zu unterschiedlichen kausalen Stärken. Dieses Argument hat freilich zwei Nachteile: Einmal ist das Problem nur verschoben, weil wir uns jetzt fragen müssen, ob zwei identische Interaktionen die gleiche kausale Stärke haben, und zum anderen hätte man bei dieser Lösung zu viele Interaktionen, als daß man damit in einem Analysemodell noch sinnvoll umgehen könnte.

Zum Glück brauchen wir uns bei dieser Unterscheidung nicht lange aufzuhalten, denn die Berücksichtigung individueller kausaler Stärken ist ohnehin utopisch, jedenfalls im Rahmen der hier untersuchten analytischen Instrumente. Notfalls - i.e. sollte die erste Position die richtige sein müBte man sich mit Durchschnittswerten begnügen, die so etwas wie die durchschnittliche Stärke der Wirkung einer kausalen Kraft angeben.

\section{Notwendige Voraussetzungen für die kausale Analyse II: Der Zustand ohne Kausalität}

Wir sind in der Diskussion der kausalen Modellwelt so weit gelangt, da B wir die ersten tastenden analytischen Schritte vornehmen können. Vieles muß dabei notwendig vorläufig bleiben, weil wir uns z.B. mit der Frage, welche kausalen Kräfte es gibt, noch gar nicht beschäftigt haben. Aber gleichwohl sind wir weit genug, uns ein Problem zu exponieren, dessen vollständige Lösung für eine kausale Analyse notwendige Bedingung ist. Für die genaue Formulierung des Problems müssen wir uns kurz an die analytische Ausgangssituation erinnern. Durch empirische Erhebung wurde ein $(n+r)$-dimensionaler Datenkubus gefunden, der den Zustand zu einem Zeitpunkt $\mathrm{T}_{2}$ darstellt. Gesucht sind die kausalen Kräfte, die von einem Zustand zu dem Zeitpunkt $T_{I}$ zu dem erhobenen Zustand geführt haben. 
An dieser Stelle erscheint es nützlich, auf die Unabhängigkeit unserer Untersuchung von konkreten Analysemodellen, wie sie von Soziologen in ihren Untersuchungen eingesetzt werden, hinzuweisen. Denn wer den bisherigen Ausführungen nur oberflächlich gefolgt ist, der könnte jetzt fragen, "Warum soll ich mich um verschiedene Weltzustände kümmern und insbesondere um solche, von denen ich doch nichts genaues wissen kann? Ich analysiere meine Daten mit den Verfahren XYZ und dann. weiB ich, welche Kausalität vorliegt, weil mein Modell mir dies so angibt." Dem kann man nur folgendes entgegenhalten: Kausalität ist (wenn man es salopp formuliert) nichts anderes als eine Veränderung von Weltzuständen durch die Wirkung kausaler Kräfte. Entweder wird dies in einem Analysemodell explizit oder implizit berücksichtigt, oder das Modell liefert keine korrekten Resultate (wobei der Fall zufällig richtiger Resultate hier ausgeschlossen werden soll).

Den Beweis für die Richtigkeit dieser Behauptung können wir sehr schnell erbringen: Er erfolgt durch eine Reductio ad Absurdum. Eine solche Reductio liegt sicher dann vor, wenn aus dem vorliegenden Datenkubus jede kausale Interpretation durch ein Analysemodell herausgezogen werden kann. Denn eine Analyse, die jedes Resultat liefern kann, wäre nutzlos, ihr Wert besteht ja gerade darin, das richtige Resultat zu finden, i.e. alle bis auf eines auszuschließen.

Der Beweis ist denkbar einfach: Man gibt sich die kausalen Kräfte mit ihren jeweiligen Stärken vor. Damit gibt man sich genau das vor, was man eigentlich herausfinden wollte und wählt dann den Weltzustand $\mathrm{T}_{\mathrm{I}_{\mathrm{n}}}$ aus, der mit diesen Verschiebungen zu dem Weltzustand $T_{2}$ führt. Dann sind genau zwei Fälle denkbar:

- einen solchen Weltzustand $T_{1}$ gibt es nicht, weil die geforderte Verschiebung aus logischen Gründen ausgeschlossen werden kann.

- einen solchen Weltzustand $T_{I}$ gibt es, weil die geforderte Verschiebung denkbar ist.

Ausgeschlossen werden kann nur die erste Klasse von Fällen, aber aus rein logischen Gründen. Von der allein für uns (qua empirische Sozialforscher) wichtigen zweiten Klasse von Fällen kann nicht ein einziges Modell ausgeschlossen werden. Wie man $T_{1}$ dabei wählt, kann man sich ungefähr so vorstellen: Man läßt in $\mathrm{T}_{2}$ die von dem Modell geforderten kausalen Kräfte 'rückwärts' wirken und hat dann die Tabelle $T_{I}$, die $z u$ $\mathrm{T}_{2}$ führt.

Damit sollte auch deutlich geworden sein, warum jedes konkrete mathematische Kausalmodell, das nicht mit allen denkbaren kausalen Interpretationen verträglich ist, zumindest implizite Vorannahmen über den Weltzustanc' $T_{1}$ macht - machen muB! -, auch wenn darüber in Beschreibungen nie 
die Rede ist. Deutlich wird aber auch, warum es methodisch unangemessen sein kann, wenn der explizite Bezug auf $T_{I}$ und $T_{2}$ vermieden wird und so bei einer flüchtigen Rezeption der Eindruck entsteht, als liefere uns ein bestimmtes mathematisches Kausalanalysemodell ein Wissen über kausale Vorgänge, das über eine mathematische Analyse nicht gewonnen werden kann. Es gibt selbstverständlich keine Möglichkeit durch einen a priori Schluß auf die Tabelle $T_{I}$ zu schließen, wenn die Tabelle $T_{2}$ vorliegt. Wenn wir ein bestimmtes $T_{1}$ annehmen, dann geht empirisches Wissen über den Weltzustand bezüglich der relevanten Variablen ein.

Um eine griffige Terminologie zu haben, bezeichnen wir eine Tabelle $T_{I}$ als Nulltabelle. Das Resultat der bisherigen Überlegungen ist, da $B$ es keine kausale Analyse geben kann, ohne daB bestimmte Nulltabellen als empirisch unplausibel ausgeschlossen werden können. Dort wo man über ein entsprechendes empirisches Wissen verfügt, etwa durch eine Erhebung zu dem entsprechenden Zeitpunkt, ist dieser Teil der Analyse unproblematisch, nur wird dieser Fall in der Praxis selten eintreten. Man hat nur das Wissen über $T_{2}$ und möchte gleichwohl eine kausale Analyse vornehmen. Der nächstliegende Schluß ist dann: $T_{I}$ soll eine Tabelle sein, in der die Wirkung der kausalen Kräfte fehlt. ${ }^{I}$ D.h. $T_{I}$ muß eine Tabelle sein, die nicht assoziiert ist. Hält man an der durch $\mathrm{T}_{2}$ gegebenen Randverteilung fest, dann ist $T_{I}$ die nicht-assoziierte $\mathrm{T}_{2}^{2}$ belle, wie wir sie aus der Berechnung von $x^{2}$ kennen.

Damit hätte man dieses Problem auf einfache, elegante und vertraute Weise gelöst. Aber leider ist die Úberlegung aus zwei Gründen nicht korrekt:

- selbst wenn man sich für eine nicht assoziierte Tabelle als Nulltabelle entscheidet, muB die Wahl nicht auf eine $x^{2}$-Indifferenztabelle fallen.

- Es ist nicht in jedem Fall einsichtig, warum eine Nulltabelle nicht assoziiert sein sollte.

Das einzige Argument für eine $x^{2}$-Indifferenztabelle als Nulltabelle stuitzt sich auf die Identität von Randverteilungen bei $T_{1}$ und $T_{2}$. Aber da die Wirkungen der Kausalität gerade darin bestehen, die Verteilung in der abhängigen Variablen zu verändern, gibt es für identische Randverteilungen bei der abhängigen Variablen keinen Grund. Hat man als Bedingungen nur "keine Assoziation", dann ist jede nicht assoziierte Tabelle eine potentielle Nulltabelle. Die $\chi^{2}$-Indifferenztabelle hat allen anderen gegenüber keinerlei Vorzüge. Wer trotzdem genau diese Tabelle als Nulltabelle wählt, muß dafür besondere Gründe angeben.

Aber warum soll $T_{I}$ nicht assoziiert sein? Die Antwort ist einfach und scheinbar zwingend: Wenn keine der unabhängigen Variablen einen kausalen Einfluß auf die aḅhängige Variable hat, dann müssen die Verteilungen 
in den Kategorien der unabhängigen Variablen identisch sein, denn genau so zeigt sich das Fehlen eines kausalen Einflusses. Es wäre m.a.W. konzeptueller Nonsens, wenn man den SchluB

$$
\text { "keine Kausalität } \rightarrow \text { keine Assoziation" }
$$

nicht als zwingend ansehen würde.

Dieses Argument wäre nur dann korrekt, wenn es nicht eine zumindest praktisch dubiose Prämisse enthielte: Der obige Schluß ist nämlich nur dann richtig, wenn alle kausal relevanten Variablen in der Analyse berücksichtigt werden. Das aber ist praktisch kaum durchfuhrbar, weil wir bei abhängigen Variablen wie "Einstellung zur Friedensbewegung" mit so vielen untereinander kausal verknüpften Variablen rechnen müssen, da $B$ es wegen der für eine vollständige Analyse erforderlichen großen Zahl von Fällen unmöglich sein dürfte, alle Variablen auf einmal zu berücksichtigen. Damit aber gilt der obige Schluß nicht mehr, denn vollständig lautet er ja:

"Wenn alle kausal wirksamen Variablen berücksichtigt sind $\rightarrow$ (keine Kausalität $\rightarrow$ keine Assoziation)".

Nun hatten wir eingangs gerade praktische Argumente, die sich auf empirisch nur sehr schwer erfüllbare Bedingungen beziehen, ausschließen wollen, so daß dieses Argument mit der gemachten Einschränkung nicht überzeugend ist, auch wenn es aus der Anwendungsperspektive zwingend erscheint. Aber es gibt auch ein mehr theoretisches Argument, das in die gleiche Richtung zielt: Wenn man eine Variable in einer Untersuchung berücksichtigt, dann geschieht das ja hauptsächlich deswegen, weil wir an die kausale Wirksamkeit dieser Variable glauben. Wenn kausale Analyse aber mehr sein soll als die Bestätigung plausibler kausaler Zusammenhänge, dann muB sie prinzipiell auch zur Aufdeckung neuer kausaler Einflüsse imstande sein. Und genau dies ist dann nicht möglich, wenn ich für die Richtigkeit der Analyse annehmen muB, daB alle kausal relevanten Variablen bereits berücksichtigt sind. In $\mathrm{T}_{\mathrm{I}}$ sind ja alle die Einflüsse von kausalen Variablen enthalten, die in der Analyse nicht berücksichtigt werden. $T_{I}$ als eine nicht assoziierte Tabelle anzunehmen müBte dann die Existenz solcher Variablen bestreiten (oder annehmen, sie würden sich in ihrer Wirkung gerade kompensieren).

Wir stoßen wieder auf ein sehr schwer auflösbares Dilemma kausaler Analysen, so wie sie von Soziologen vorgenommen werden. Einerseits wird man alle kausal relevanten Variablen in die Untersuchung einbeziehen und demnach eine nicht assoziierte Nulltabelle als die Beschreibung des Zustandes ohne Kausalităt wählen. Andererseits wissen wir aus abstrakten Überlegungen, daB es uns unbekannte (z.B. theoretische) Variablen geben wird, die nicht in der Analyse vorkommen. Deshalb ist die Annahme einer nicht as- 
soziierten Kontingenztabelle falsch und demnach können die Resultate der Analyse nicht korrekt sein.

Das alles wäre nicht weiter störend, wenn man den Fehler nach der Analyse bemerken würde, aber eben dies ist nicht der Fall. Um das zu sehen, mu $B$ die Situation etwas genauer analysiert werden. Wir müssen unterscheiden $z$ wischen

- der wahren Nulltabelle $T_{\text {. }}$. (Ich hoffe, daß der Leser sich spätestens hier an den vielfältigen Gebrauch des Symbols ' $\mathrm{T}$ ' gewöhnt hat.) Sie enthält den Zustand ohne Kausalităt bezüglich der explizit in der Analyse berücksichtigten Variablen. Aber sie enthält auch alle kausalen Effekte, die durch Variablen bewirkt werden, die mit den explizit berücksichtigten Variablen kausal verknüpft sind. Damit wird die Nulltabelle $T_{I}$ i.a. eine assoziierte Tabelle sein. Das liegt daran, $\mathrm{da} B$ bei Untersuchungen wie der hier diskutierten eine große Zahl von empirisch und theoretisch kausal relevanter Variablen vorliegt, die mit den berücksichtigten kausal verknüpft sind. Diese für kausale Analysen so unangenehme Konsequenz ist die Grundlage für die Soziologen-Weisheit, derzufolge "alles mit allem zusammenhängt".

- der fälschlich angenommenen Nulltabelle $T_{I}$. Sie ist nicht assoziiert.

- den korrekten Verschiebungen von $T_{1}$ zu $T_{2}$. Korrekt sind dabei die Verschiebungen, die in der Realität vorliegende Kausalităt simulieren.

- der Verschiebung von $T_{I}^{\prime}$ zu $T_{2}$. Sie wird möglicherweise von den gleichen Variablen bewirkt, wie die zuvor genannten Verschiebungen, aber diese können wegen der Differenz von $T_{I_{1}} z_{u_{1}} T_{1}$ nicht die richtige Größe haben.

Unproblematisch wăre der Fehler, den wir machen, indem wir $T_{\mathrm{I}}$ durch $T_{1}^{\prime}$ ersetzen, nur dann, wenn wir dies durch eine mangelnde Anpassung bemerkten. Wir versuchen von $T_{1}^{\prime} \mathrm{zu}_{2} \mathrm{zu}$ gelangen und erreichen nur eine höchst mangelhafte Anpassung. Aber dies ist nicht der Fall, weil man bei einer großen Klasse von Fällen auch dann hinreichend gute Anpassungen erreicht, wenn von falschen Nulltabellen ausgegangen wird.

Dies ist bei einer experimentellen Überprüfung kausaler Kräfte nicht möglich. Denn dort können wir nicht einen Zustand $T_{I}^{\prime}$ in einen Zustand $T_{2}$ überführen, wenn es nicht Ursachen mit den entsprechenden Wirkungen gibt. Die Natur (oder die soziale Realität) versagt uns ganz einfach diesen Dienst, so da B wir sehr schnell die Insuffizienz unseres Ansatzes bemerken. Damit soll nicht gesagt werden, die Uberprifiung von naturwissenschaftlichen Theorien sei grundsätzlich einfach und Fehler würden sich 
sofort herausstellen. Tatsächlich muß man häufig einen enormen experimentellen Aufwand treiben, um eine bestimmte Hypothese überprüfen zu können. "Ganz einfach" soll hier besagen "methodisch einfach", weil wir jeweils überprüfen können, ob die postulierte Kausalität so wirkt, wie wir uns dies vorgestellt haben. Es gehört gleichsam zur Natur der Kausalităt, da $B$ die Wirkung durch Herbeiführung der Ursache erzielt werden mu $B$. Bleibt sie aus - und es lagen keine störenden Faktoren vor - so kann der behauptete kausale Zusammenhang nicht existieren. Mit Experimenten lassen sich solche Aussagen überprüfen.

Ganz anders ist es bei einer nicht-experimentellen Tabellenanalyse. Wir haben dort eine solche Auswahl von potentiellen kausalen Effekten vorliegen, $\mathrm{da} B$ wir von jeder möglichen Nulltabelle $\mathrm{T}_{1}^{\prime} \mathrm{zu}$ der vorliegenden Tabelle $\mathrm{T}_{2}$ gelangen können. Man beachte die Umkehrung der Argumentation zu dem vorher Gesagten. Dort argumentierten wir, es ließe sich immer eine Nulltabelle $T$ finden, mit der sich beliebig vorgegebene kausale Effekte herstellen ließen. Jetzt sagen wir umgekehrt, jede beliebige Nulltabelle läßt sich in die Tabelle $T_{2}$ überführen, weil hinreichend viele potentielle Ursachen zur Verfügung stehen. Wann dieses mathematisch banale aber inhaltlich kuriose Phänomen möglich ist, werden wir erst in dem nächsten Abschnitt genau untersuchen. Hier soll nur auf die wichtigen Folgen für die Möglichkeiten einer kausalen Analyse hingewiesen werden, die sich aus den Schwierigkeiten ergeben, die korrekte Nulltabelle zu finden. Man könnte in diesem Zusammenhang auch von einem geradezu bösartigen Zirkel reden: Solange wir die tatsächlichen kausalen Beziehungen nicht kennen, können wir nicht die endgültige Form der Nulltabelle $T_{I}$ festlegen. Mit einer falschen Nulltabelle können wir aber die richtigen kausalen Beziehungen nicht finden. M.a.W.: Wir können die tatsächlichen kausalen Beziehungen nur dann feststellen, wenn wir sie bereits kennen. Damit wird aber kausale Analyse als ein erkenntniserweiterndes Analyseverfahren uninteressant.

Die Schwierigkeiten dieses Verfahrens liegen u.a. auch in der Unzahl potentieller kausaler Effekte, die in einer Analyse vorkommen können. Wir haben gleichsam immer die analytischen Instrumente, um jede angepeilte Anpassung der Tabellen $T_{1}$ und $T_{2}$ zu erzwingen und werden dabei mit kausalen Verschiebungen arbeiten, die in der Realität gar nicht vorkommen. Welche potentiellen Effekte dabei zur Verfügung stehen, soll in dem nächsten Abschnitt untersucht werden. Dabei geht es nicht nur um die Abwicklung einer einfachen Zählaufgabe, sondern um sehr viel mehr: Denn welche Variablen überhaupt als potentielle kausale Ursachen für die Verschiebung in Frage kommen, ist alles andere als trivial. Da diese eher inhaltliche Frage in der technischen Literatur ubergangen wird, lohnt sich eine gründliche Diskussion, weil so auch von ganz anderer Seite her die unüberwindlichen Probleme deutlich werden, die mit kausalen Analysen verbunden sind. 
6. Notwendige Voraussetzungen für die kausale Analyse III: Die potentiellen kausalen Effekte

Das in der Uberschrift formulierte Problem hat mehrere Komponenten, die wir bei der Diskussion sorgfältig trennen müssen. Einmal kann man die Frage im Sinn von: "Wieviele potentielle kausale Effekte gibt es?" verstehen. Die Antwort darauf ist ein Abfallprodukt der Antwort auf eine weitaus wichtigere Frage: "Welche inhaltlichen Voraussetzungen müssen vorliegen, damit von einem potentiellen kausalen Effekt gesprochen werden kann?" Daran schlieBt sich eine dritte, von uns nur zu streifende Frage an: "Mit welcher Art von kausalen Effekten wird man es typischerweise in der Soziologie zu tun haben?"

In Veröffentlichungen über kausale Analysen werden wir häufig Variablen wie "Geschlecht", "Alter", "Bildung" als unabhängige "kausal erklärende" Variablen finden, und es wäre nicht verwunderlich, wenn auch in einer Studie über die Friedensbewegung diese Variablen auftauchen würden. Nun haben wir natürlich keine experimentellen Möglichkeiten, die Richtigkeit entsprechender Behauptungen zu uberprüfen, aber man kann immerhin fragen, ob die unterstellten kausalen Zusammenhänge plausibel sind. Angenommen, wir finden die Behauptung "Alter ist eine wesentliche Ursache für die Beteiligung an der Friedensbewegung", wobei im Detail ausgeführt wird, da $B$ insbesondere junge Leute sich an der Friedensbewegung beteiligen. Im Sinne der vorherigen Ausführungen müBte dies so interpretiert werden: "Alter" ist eine kausale Kraft, die von "friedensbewegt" zu "nicht friedensbewegt" verschieben kann. "Alter" ist demnach ceteris paribus eine hinreichende Bedingung für diese Verschiebung. Dies kann man auch so formulieren: Gleichgültig, welche anderen Eigenschaften jemand noch hat, allein das Alter wird eine entsprechende kausale Kraft ausüben. (Natürlich dürfen wir nicht ohne weiteres annehmen, die entsprechende Verschiebung komme auch zustande, denn es kann ja entgegenstehende kausale Kräfte geben.)

Damit ist deutlich, welche Variablen oder Interaktionen wir für die Verschiebungen in dem Datenkubus benötigen. Sie müssen ceteris paribus (i.e. solange keine anderen kausalen Kräfte dem entgegenstehen) für die Verschiebung hinreichend sein. Ein Gedankenexperiment macht den entscheidenden Punkt drastisch deutlich: Die Situation ist etwas komplizierter als es hier dargestellt wird, weil man im alltäglichen wie wissenschaftlichen Kontext sich nicht für hinreichende kausale Bedingungen interessiert, sondern nur für jene kausalen Bedingungen, die zusammen mit anderen, deren Erfülltsein als selbstverständlich vorausgesetzt wird, hinreichend sind. Für die vorgetragene Kritik ist diese Unterscheidung freilich unwichtig, so da B sie im folgenden nicht getroffen wird. Leider ist es bei vielen als kausal relevant eingestuften Variablen nicht der Fall, daß sie für eine Verschiebung hinreichend sind, oder zumindest ist es nicht offensichtlich. In dem 
obigen Beispiel wurde gesagt, Alter sei die wesentliche Ursache für die Beteiligung an der Friedensbewegung. Demnach müBte ein Mensch (mit einer bestimmten Wahrscheinlichkeit, wenn es um probabilistische Kausalität geht) einfach durch Altern von dem Zustand "friedensbewegt" in den $\mathrm{Zu}$ stand "nicht friedensbewegt" übergehen. Vermutlich dürfte aber niemand glauben, daß Altern allein die Ursache für eine solche Entwicklung sein kann, vielmehr kommen andere Faktoren hinzu, wie die Veränderung der Interessen durch die berufliche Arbeit, neue soziale Umfelder etc. Es kann hier nicht darum gehen, die genauen Zusammenhänge zwischen Altern und dem Engagement in der Friedensbewegung herauszuarbeiten, die sind uns ebenso unbekannt wie diejenigen, die mit diesen Variablen arbeiten. Ziel ist allein die Problematisierung allzu einfacher kausalanalytischer Annahmen, denen zufolge einzelnen Variablen eine kausale Wirksamkeit unterste!lt werden kann. Wir wissen einfach nicht, ob Alter eher friedlich oder eher unfriedlich macht, und vor allen Dingen wissen wir nicht, welche anderen Kausalfaktoren noch mit hinzukommen müssen, damit Altern die unterstellte kausale Wirksamkeit entfalten kann.

Hier stoßen wir wieder auf jenen unfruchtbaren Zirkel, nach dem erst dann sinnvoll Kausalanalyse betrieben werden könnte, wenn unser Wissen über kausale Gesetze der sozialen Realität sehr viel größer wäre. Andererseits versuchen wir eben dieses Wissen durch die geschilderten insuffizienten Verfahren zu ergänzen, was nicht gelingen kann.

Was hier an der Variable "Alter" diskutiert wurde, gilt auch für die Variable "Geschlecht" und sehr viele der in kausalen Analyse verwendeten Variablen. Selbst wenn es richtig sein sollte, daß Frauen eher der Friedensbewegung angehören als Männer, so dürften es kaum die biologischen Unterschiede sein, die diesen Unterschied bedingen. Abermals macht ein Gedankenexperiment den entscheidenden Punkt drastisch deutlich: Durch eine Geschlechtsumwandlung allein würde wohl niemand seine Einstellung zur Friedensbewegung ändern. Deshalb kann Geschlecht keine Ursache für eine kausale Verschiebung sein. Eher denkt man an unterschiedliche soziale Rollen, unterschiedliche soziale Wahrnehmungen, so daß es primär der soziale Unterschied zwischen Frauen und Männern ist, der die unterschiedliche Affinität zur Friedensbewegung erklärt. Dieser soziale Unterschied ist eine nur durch viele Variablen (und deren komplexes kausales Geflecht) erklärbarer Zusammenhang, so daß die Behauptung, "Geschlecht" sei eine kausal relevante Variable, allenfalls in diesem allgemeinen Sinn richtig wäre - obwohl die Redeweise nicht besonders glücklich ist.

Was wir suchen sind Ursachen, die - ceteris paribus - für eine Verschiebung hinreichend sind. Mit den üblichen Variablen in der soziologischen Kausalanalyse sind solche Ursachen nicht identifiziert. Variablen wie "Alter", "Geschlecht", "Bildung", "sozialer Status" etc. sind deswegen nicht kausal irrelevant, nur sind sie auf vielfältige und unübersichtliche 
Weise mit anderen Variablen verknüpft, so da $B$ einfach nicht gesagt werden kann, sie hătten die geforderte Eigenschaft. Deshalb ist der näherliegende Gedanke, die für diese Verschiebung hinreichenden Bedingungen zu präzisieren, indem man von einer Variablen zu Interaktionen übergeht. Eine Interaktion von $\mathrm{n}$ Variblen (richtiger wäre: von den durch die Ausprägung bezeichneten Ereignissen) liegt genau dann vor, wenn diese $n$ Variablen zusammen eine Verschiebung bewirken, die nicht bereits durch eine Teilmenge der daran beteiligten Variablen bewirkt wird. Interaktionen sind aus zwei Gründen aussichtsreiche Kandidaten bei der Suche nach hinreichenden Bedingungen für kausale Verschiebungen:

- Zum einen war die Suche bei einfachen Variablen erfolglos, so da B es nur Interaktionen sein könnten, wenn es überhaupt identifizierbare Ursachen in der kausalen Modellwelt geben soll.

- Zum anderen hat gerade die reasons and causes Debatte mit ihren detailliert ausgeführten Beispielen gezeigt, wie komplex die Ursachen für einzelne Ereignisse sind. Das stützt die Vermutung, da B nur in dem Zusammenspiel sehr vieler Variablen Ursachen für soziale Ereignisse vorliegen können.

Interaktionen haben gegenüber Variablen einen entscheidenden Nachteil, es sind ihrer nämlich zu viele. Betrachten wir den Fall einer. Analyse mit ro unabhängigen und einer abhängigen Variablen. Eine Interaktion erster Ordnung liege dann vor, wenn ein Ereignis (i.e. eine Verschiebung) durch ein Zusammenwirken zweier Variablen eintritt, allgemeiner reden wir von einer Interaktion ( $n-r)$ ter Ordnung, wenn für ein Ereignis das Zusammenwirken von $n$-Variablen notwendig ist. Bei ro unabhängigen Variablen gibt es demnach:

$$
\begin{aligned}
& \left(\begin{array}{c}
10 \\
2
\end{array}\right) 2^{2} \text { Interaktionen erster Stufe } \\
& \left(\begin{array}{c}
10 \\
3
\end{array}\right) 2^{3} \text { Interaktionen zweiter Stufe } \\
& \cdot \\
& \cdot \\
& \cdot \\
& \cdot \\
& \left(\begin{array}{l}
10 \\
10
\end{array}\right) 2^{10} \text { Interaktionen neunter Stufe }
\end{aligned}
$$

Dies summiert sich zu einigen tausend Interaktionen. Vielleicht ist das der pragmatische Hintergrund für die Unlust von Kausalanalytikern, sich mit Interaktionen und insbesondere mit Interaktionen sehr hoher Stufe abzugeben. Trotzdem haben gerade hohe Interaktionen - also Interaktionen, bei 
denen sehr viele Variablen zusammenwirken - den Vorzug einer hochgradigen Spezifikation von Ursachen, und ihre Verwendung ist damit zunächst einmal nicht dem Einwand ausgesetzt, der gegen die Verwendung von einfachen Variablen formuliert wurde: $\mathrm{DaB}$ sie nämlich kausal etwas mit der Verschiebung zu tun hätten, aber eben nur in Zusammenhang mit anderen Variablen.

Das Argument läßt sich noch verstärken: Wenn eine Verschiebung überhaupt eine andere Ursache als eine Variable hat, dann muß diese Ursache als eine Interaktion formulierbar sein, denn eine andere Möglichkeit gibt es nicht. Man könnte einige der handlungstheoretischen Diskussion entlehnte Beispiele anführen, die eindringlich deutlich machen, wie komplex Ursachen/Gründe von Handlungen sind. So wird vielleicht jemand 'sich der Friedensbewegung anschließen, weil er bei der Stationierung neuer Mittelstreckenraketen die Kriegsgefahr drastisch wachsen sieht, weil er glaubt, da B keine der etablierten Parteien in der Lage ist, dagegen etwas zu tun, weil er nur durch Teilnahme an der Friedensbewegung eine Chance zur Veränderung der Stationierung sieht ... Es fällt nicht sonderlich schwer, sich eine große Zahl von notwendigen Bedingungen auszumalen, die zusamen hinreichend für eine Beteiligung an der Friedensbewegung sind und so die Dringlichkeit der Berücksichtigung hoher Interaktionen in der Kausalanalyse begründen. Zu bedenken ist allerdings der Endpunkt einer Ausdifferenzierung aller kausal relevanten Ursachen einer Handlung. Zumindest in einer Variante gelangt man dann zu einer Menge von Prämissen, die die Verknüpfung von Prämissen (den Ursachen der Handlung) und Konklusion (dem Satz, in dem die Handlung beschrieben wird) logisch zwingend machen. Kritiker sehen in einem so ausgeführten praktischen Syllogismus ein starkes Argument gegen eine kausale Deutung der Beziehung zwischen Prämissen und Konklusion und möchten deswegen nicht von Ursachen (causes), sondern von Gründen (reasons) reden. Damit hätten wir ein kurioses Resultat erzielt, wenn man an die Forschungsinteressen einer kausalen Soziologie denkt. Ausgangspunkt war die Uberlegung, Interaktionen seien besonders geeignete Kandidaten für die Analyse multivariater Tabellen, und der Endpunkt des Gedankens ist die Feststellung, da B zwischen dem-unabhängigen und dem abhängigen Ereignis gar keine kausale Relation besteht!

So drastische SchluBfolgerungen mu B man nicht ziehen, weil sich Vereinbarkeitsthesen (zwischen reasons and causes) formulieren lassen, aber trotzdem sollte auch dies eine deutliche Warnung vor einem leichtfertigen Umgang mit Ursachen in kausalen Analysen sein. Für welche Position man sich in dieser handlungstheoretischen Debatte entscheiden soll und welche Auswirkungen dies für kausale Analysen in den Sozialwissenschaften hat, kann hier nicht untersucht werden, weil das weit über den Rahmen dieser Arbeit hinausginge. Soviel allerdings läßt sich begründet vermuten: Eine Berücksichtigung dieser Diskussionslinie würde weitere Argumente gegen 
die Möglichkeit einer kausalen Tabellenanalyse liefern und das in diesem Aufsatz skizzierte Bild nochmals verkomplizieren. Darauf verzichten wir auch deswegen, weil es auch so hinreichend gute Gründe für die Grundthese dieser Arbeit gibt.

Will man die drastische SchluBfolgerung vermeiden und als Konsequenz aus dem Einwand Kausalanalyse mit hohen Interaktionstermen betreiben, dann steht man vor einem praktisch unlösbaren Problem. Denn wegen der riesigen Zahl von hohen Interaktionstermen müssen fast alle als kausal irrelevant ausgeschlossen werden können. Wir müssen demnach vor der Analyse bereits wissen, welche Interaktionen keine verschiebende Wirkung haben können. Bei einigen wenigen mag man dies plausibel vermuten können, aber es müssen weitaus mehr Interaktionen ausgeschlossen werden; damit die Modelle nicht hoffnungslos überbestimmt sind. Und dafür müßten wir auf ein kausales Wissen zurückgreifen, das wir nicht haben.

Der Leser wird richtig vermuten, wie jetzt weiter argumentiert wird: Es liegt wieder der bereits in den beiden vorherigen Abschnitten erwähnte Zirkel vor. Um kausalanalytisch sinnvoll vorgehen zu können, brauchen wir die Interaktionen, aber um $\mathrm{zu}$ wissen, welche Interaktionen man berücksichtigen soll, braucht man kausales Wissen. So bekommt man das eine wieder nicht, weil man das andere nicht hat und umgekehrt.

So bleibt nur die resignative Schlußfolgerung, daß trotz einiger Argumente für die Wichtigkeit auch hoher Interaktionen in kausalen Analysen und trotz der methodischen Insuffizienz von Variableneffekten eine begründete Berücksichtigung von Interaktion nicht möglich ist. Die Frage, "Was sind die potentiellen Effekte einer kausalen Analyse?" ist zuvor in dem Sinne beantwortet worden, da B wir auf die Relevanz von Interaktionen hinweisen konnten, wenn dies auch leider ohne praktische Anwendung bleibt.

\section{Eine weitere notwendige Voraussetzung jeder kausalen Analyse in der Soziologie}

In der bisherigen Argumentation haben wir auf jede detaillierte Auseinandersetzung mit bestimmten kausalanalytischen Analysemodellen verzichtet. Unsere Strategie war es vielmehr, jene impliziten Annahmen zu untersuchen, die bei jeder Kausalanalyse gemacht werden müssen, ganz gleichgültig, ob man sich in der Formulierung darauf explizit bezieht oder nicht. Drei Annahmen wurden dabei herausgearbeitet:

I) Annahmen über die Stärke kausaler Effekte: Diese Annahmen sind notwendig für die Bilanzierung der Verschiebungskräfte und damit für die korrekte Simulation tatsächlich kausaler Verschiebungen. Fehler bei diesen Annahmen führen zu falschen Verschiebungen/Festhaltungen, 
i.e. Individuen werden verschoben, die nicht verschoben werden dürfen und umgekehrt.

2) Annahmen über die Form der Nulltabelle: Die Nulltabelle ist die Ausgangstabelle für simulierte kausale Verschiebungen. Da die Verschiebungen jeweils dazu dienen, möglichst dicht an die empirisch gewonnene Tabelle heranzukommen, impliziert eine inkorrekte Nulltabelle Fehler bei Art und Stärke der gefundenen kausalen Kräfte.

3) Annahmen über die potentiellen kausalen Effekte: Die kausalen Effekte werden durch das konkrete Kausalmodell festgelegt, mit dem eine Analyse gerechnet wird. Man mu $B$ wegen des Problems der Überbestimmtheit vor einer Analyse wissen, welche Variablen/Interaktionen als Ur sachen einer Verschiebung nicht infrage kommen. Ohne dieses Wissen kann eine Analyse ebenso grob fehlerhaft werden, wie bei den Defiziten in $(\mathrm{I})$ und $(2)$.

Die Diskussion dieser drei Probleme führte jeweils auf einen Zirkel in dem folgenden Sinn: Um die für eine kausale Analyse notwendigen Annahmen begründen zu können, müBte man bereits über ein kausales Wisssen verfugen, das wir aber gerade durch solche Analysen erst erwerben wollen. Diese Form der Argumentation ist einem naheliegenden Einwand ausgesetzt: Man könnte nämlich ein ähnliches Argument verwenden, um zu begründen, warum es auch in den Naturwissenschaften nie ein kausales Wissen hätte geben können. Denn, so ließe sich analogisierend sagen, auch bei kontrollierten Experimenten machen wir von kausalem Wissen Gebrauch, weil eine Kontrolle sonst gar nicht möglich wäre. Somit könnte es auch kein naturwissenschaftliches kausales Wissen geben, weil man die Experimente nur durchführen kann, wenn das Wissen, das mit ihnen erworben werden soll, bereits vorliegt. Da es dieses Wissen gibt, muB das Argument inkorrekt sein und -wegen der Analogisierung - auch sein soziologisches Pendant.

Diese Argumentation ist aber falsch, weil sie die Möglichkeit einer ständigen Kumulation kausalen Wissens außer Acht läßt. Indem wir mit einem einfachen, alltäglichen kausalen Wissen beginnen, können wir Schritt für Schritt zu immer komplexeren kausalen Gesetzmäßigkeiten vordringen und so den Zirkel in eine Spirale auflösen.

Als grobe Näherung ist diese Beschreibung für die Naturwissenschaften richtig, und warum sollte sie nicht auch für die Soziologie gelten? Dabei muß zwischen zwei Fragen unterschieden werden:

I) Ist eine solche Entwicklung denkbar, oder muB sie aus prinzipiellen Gründen ausgeschlossen werden?

2) Wenn eine solche Entwicklung möglich ist, welche Beiträge können dabei bekannte Kausalanalyseverfahren leisten? 
Die Diskussion der zweiten Frage führt uns auf eine äußerst wichtige weitere notwendige Voraussetzung für Kausalanalyse in der Soziologie. Wir unterstellen einfach eine positive Antwort auf die erste Frage, um die potentiellen Beiträge găngiger Kausalanalyseverfahren bei der Kumulierung kausalen Wissens einschătzen zu können.

Unterstellen wir also, wir wüBten

- von einigen Interaktionen, daB sie kausale Verschiebungen von friedensbewegt $\mathrm{zu}$ nicht friedensbewegt (oder umgekehrt) bewirken und von einigen hundert Interaktionen, daB sie kausal irrelevant sind;

- wie stark die einzelnen kausalen Kräfte sind;

- wieviel Prozent aller Individuen von den jeweiligen kausalen Verschiebungen betroffen sind;

- die ungefähre Form der Nulltabelle.

Wie weit dieses Wissen im einzelnen geht, ist jetzt nicht interessant. Warum ist dieses Wissen so extrem wichtig für eine mögliche Kumulation kausalen Wissens in der Soziologie? Um das zu begründen, knüpfen wir an die drei Zirkel der vorherigen Abschnitte an. Das Problem dort war jeweils gewesen, da B man ein bestimmites Wissen haben muB, um Kausalanalyse betreiben zu können, aber andererseits jenes Wissen mit dieser Kausalanalyse erst gewinnen kann. Wenn nun aber ein kausales Wissen bereits verfügbar ist, dann ist ein Auflösen des Zirkels in eine Spirale zumindest denkbar. Aber dazu muB dieses Wissen als Vorwissen in die Analyse einbezogen werden können.

Man kann das Problem auch etwas formaler behandeln und fragen, was denn die drei erwähnten Zirkel in formaler Hinsicht so bedrohlich macht. Warum ist denn eine kausale Analyse nicht möglich, wenn ich

- nichts oder zuwenig über die Nulltabelle weiß,

- nichts oder zuwenig über die möglichen kausalen Effekte weiß,

- nichts oder zuwenig über kausale Stärken weiB?

Die Antwort mag auf den ersten Blick paradox erscheinen: Weil wir dann viel zu viele Möglichkeiten haben, um eine perfekte Anpassung von $T_{\mathbf{I}}$ an $T_{2}$ zu erreichen. Das mag deshalb paradox klingen, weil man antworten möchte, daß eine perfekte Anpassung doch die beste mögliche Anpassung sei. Ja, aber man hat nichts davon, wenn man bei einer Unzahl von einander ausschlieBenden Modellen eine solche Anpassung erzielen kann. Durch die große Anzahl von Freiheitsgraden kann man - etwas vereinfacht ausgedrückt - jedes kausale Modell bestätigen. Aber dann kann man gleich ganz auf die Analyse verzichten. 
Man kann das Problem einer zu großen Anzahl von Freiheitsgraden nur durch den Einbau kausalen Wissens in dem obigen Sinne in den Griff bekommen. Denn je stärker die Randbedingungen sind, die wir zu berücksichtigen haben, desto kleiner wird der Spielraum für mögliche Modelle.

Damit haben wir eine weitere äußerst wichtige notwendige Bedingung für eine inhaltlich triftige Kausalanalyse in der Soziologie gefunden: Analysemodelle müssen relevantes Vorwissen als Randbedingung in die Analyse integrieren können. Dies ist nicht nur aus technischen Gründen zwingend geboten, es ist auch eine inhaltlich unverzichtbare Forderung: Kumulativer Wissenserwerb verlangt ja gerade, $\mathrm{da} B$ das neu gefundene in das bereits vorhandene Wissen integriert werden kann. Dabei steht das bereits vorhandene Wissen nicht zur Disposition. Eine wissenschaftliche Disziplin kann eben nicht immer wieder bei Adam und Eva beginnen.

Inwieweit sind gängige Kausalanalyseverfahren geeignet, einen kumulativen Wissenserwerb zu unterstützen? Oder, deutlicher formuliert: Inwieweit genügen găngige Kausalanalyseverfahren der Voraussetzung, ohne die kausale Analyse als wissenschaftlicher Proze B nicht denkbar ist? Wir sehen bei der Diskussion über die erläuterten Schwachstellen üblicher Modelle hinweg, wichtige kausale Parameter nicht explizit zu berücksichtigen und beschränken uns auf die Stärke einzelner kausaler Effekte, i.e. auf Aussagen des folgenden Typus:

- 'Frau' ist in $43 \%$ aller (möglicher Fälle) die Ursache für die Verschiebung von nicht friedensbewegt zu friedensbewegt.

- Die Interaktion aus hoher Bildung und Jugend ist in $70 \%$ der möglichen Fälle hinreichend für die Verschiebung von nicht friedensbewegt zu friedensbewegt.

- Der Sozialstatus des Vaters ist für die Einstellung zur Friedensbewegung kausal irrelevant.

- Keine Interaktion von höherer als 4ter Ordnung ist für die Einstellung zur Friedensbewegung kausal relevant.

Wenn wir von der Richtigkeit entsprechender Behauptungen wüßten und wollten sie als Randbedingung in der kausalen Analyse verarbeiten, in welcher Weise würden uns gängige Modelle für die Analyse nominal skalierter Daten (z.B. log-lineare Modelle) dabei unterstützen? Möglich ist dies nur bei den beiden letzten Bedingungen: Wenn der Sozialstatus des Vaters für die Analyse kausal irrelevant ist, dann wird man ihn in dem entsprechenden Modell nicht berücksichtigen. Ebenso wird man keine Interaktion von höherer als 4ter Ordnung berücksichtigen, wenn all diese Interaktionen für die Einstellung zur Friedensbewegung irrelevant sind. 
Man kann diese Möglichkeit, kausale Effekte zu berücksichtigen, auch so charakterisieren: Kausal nicht relevante Effekte werden einfach aus dem Modell weggelassen. In unserer Terminologie könnte man den gleichen Sachverhalt so formulieren: Da kausal nicht relevante Effekte keine Verschiebung bewirken können, kann man sie unberücksichtigt lassen, oder etwas umständlicher formuliert: Kausal nicht relevante Effekte werden mit einer Verschiebung von Null berücksichtigt.

Diese zunächst sehr merkwürdig klingende Formulierung macht deutlich, da $B$ wir in gängigen Kausalanalysemodellen denkbare Randbedingungen berücksichtigen können. Aber eigenartigerweise gestatten gängige Modelle nur die Wahl zwischen den beiden folgenden Möglichkeiten:

- ein Effekt wird in der Analyse berücksichtigt und hat dann den durch das Modell geschätzten Wert;

- ein Effekt wird nicht berücksichtigt und hat demnach den Wert Null.

Damit ist es aber nicht möglich, Informationen über kausale Zusammenhänge zu berücksichtigen, wie sie durch die ersten beiden hypothetischen Aussagen angegeben wurden. Wenn wir aus einer vorläufigen Untersuchung wissen, $\mathrm{da} B$ hohe Bildung und Jugend eine Interaktion mit einem bestimmten Wert bilden, dann ist es notwendig, diese von uns als sicher angesehene Information als Randbedingungen in weiteren Analysen verwenden zu können. Denn immer dann, wenn dieser kausale Effekt als Randbedingung in die Analyse eingeht, verändert er indirekt andere kausale Effekte, weil bei der Anpassung der Nulltabelle an die erhobene Tabelle die bereits vorgenommene Verschiebung berücksichtigt wird.

Warum ist es so eigenartig, wenn gängige Modelle bei der Berücksichtigung kausalen Wissens als Randbedingung so unflexibel sind? Einfach deswegen, weil eine Kumulation kausalen Wissens in der Soziologie notwendig die Möglichkeit voraussetzt, bereits vorhandenes Wissen als Basis für die Suche nach neuen kausalen Zusammenhängen zu beutzen. Dies ist nicht nur inhaltlich plausibel, es wird auch formal durch die Notwendigkeit, die extreme Zahl von Freiheitsgraden bei einer Tabellenanalyse zu reduzieren, erzwungen. Man sollte nun eigentlich glauben, daß die Kongruenz von technischen und inhaltlichen Forderungen zu einer Proliferation von Modellen fuhrt, die in hoch flexibler Weise die Integration bereits vorhandenen kausalen Wissens im Sinne einer Randbedingung gestatten. Stattdessen verschlieBt man in der Modellbildung weitgehend die Augen vor diesem fundamentalen Problem jeder nicht experimentellen kausalen Analyse und tut so, als könne man mit jeder neuen Datenanalyse die kausale Welt neu entdecken. 
Das ist deswegen so kurios, weil mit dieser Vorgehensweise ein stetiger kumulativer Ausbau kausalen Wissens selbst dann nicht vorstellbar ist, wenn alle anderen kritischen Einwände nicht gelten würden. Die mathematischen Modelle sind von vornherein für die vorgesehenen Zwecke wenig geeignet, weil sie die Entwicklung, die sie angeblich mit vorantreiben sollen, wegen inhärenter Defizite gar nicht vorantreiben können. Der Leser möge diese Passagen nur nicht als eine Art naiven Vertrauens in bessere mathematische Modelle mißverstehen. $\mathrm{Ob}$ es überhaupt bessere Modelle geben kann, welche mathematische Gestalt sie haben und welche inhaltlichen Vorteile man sich davon erhoffen kann, braucht uns hier gar nicht zu interessieren. Uns interessiert allein der bemerkenswerte Umstand, daB die gängige Art mathematischer Modellbildung nicht zu den wissenschaftlichen Ansprüchen paßt, die damit eingelöst werden sollen.

Was wir notwendig bei jeder angenommenen mathematischen Modellbildung für kausalanalytische Zwecke benötigen, ist die Möglichkeit, bereits vorhandenes kausales Wissen als Randbedingung zu integrieren. Neben den bereits angedeuteten Randbedingungen sind noch weitere Typen von Randbedingungen denkbar. Wir nennen nur einige Beispiele:

- Aussagen über Bereiche in denen kausale Effekte liegen: "Bildung verschiebt zwischen 10 und $25 \%$ von nicht friedensbewegt zu friedensbewegt".

- Aussagen über die mögliche Unter- und Obergrenze: "Bildung bewirkt Verschiebungen von minimal $8 \%$ von nicht friedensbewegt zu friedensbewegt", oder "Bildung bewirkt Verschiebungen von maximal $50 \%$ von nicht friedensbewegt zu friedensbewegt".

- Aussagen über die relative Stärke von Effekten zueinander: "Die Stärke der Verschiebung von Bildung ist zumindest doppelt so gro $B$ wie die Stärke der Verschiebung von Jugend", oder "Bildung bewirkt bei zumindest $25 \%$ mehr Fällen eine Verschiebung als Jugend".

Welche Randbedingungen man wählen wird, hängt dabei allein von der Qualität des kausalen Wissens ab. Berücksichtigt man die anderen in der Arbeit beschriebenen Freiheitsgrade (Nulltabelle und Kraft einzelner kausaler Wirkungen), dann kann man sich eine sehr große Zahl möglicher Randbedingungen denken, deren Berücksichtigung für eine Analyse interssant sein könnte.

Mit der Realisierung von mathematischen Modellen, die eine Integration derartiger Randbedingungen gestatten, würde freilich nicht mehr bereitgestellt als eine Klasse von Modellen, die notwendige Voraussetzungen für eine soziologische kausale Analyse erfüllen. Man könnte sich bei der Verwendung solcher Modelle eine Kumulation kausalen Wissens durch das $\mathrm{Zu}$ - 
sammenspiel von Analyse und Datenerhebung zumindest denken. Weitere Schlußfolgerungen dürfen aus diesen Erörterungen nicht gezogen werden. In gar keinem Fall darf man sie als eine konditionale Behauptung der Art "Wenn wir diese Modelle hätten, dann könnten wir akzeptable soziologische Kausalanalyse betreiben" lesen, denn diese Behauptung ist aller Wahrscheinlichkeit nach falsch. Vielmehr sollte man die Überlegungen über angemessene Strukturen mathematischer Modelle als eine radikale Kritik an gängigen Formen der Analyse nominalskalierter Daten auffassen. Neben allen bereits erwähnten Defiziten fehlt es auch an der schlichten technischen Möglichkeit, vorhandenes kausales Wissen zu berücksichtigen und die Zahl der Freiheitsgrade in der Analyse zu verringern.

8. Theoretische Kritik und praktische Vernunft: Sind statistische Kausalanalyseverfahren besser als sie im Lichte unserer Kritik erscheinen?

Eine erfolgreiche Praxis ist allemal ein besseres Argument als es jede im Detail noch so überzeugende aber letztlich brotlose theoretische Kritik sein kann. Was wir bisher vorgetragen haben, läßt sich vielleicht griffig als ein 'no-go-Theorem' bezeichnen. Wir haben uns notwendige Bedingungen für soziologisch brauchbare Kausalanalysen überlegt und darauf hingewiesen, da $B$ jedenfalls die gängigen Verfahren zur Analyse nominalskalierter Daten diesen Bedingungen nicht oder nur unzureichend genügen. Da diese Verfahren aber tausendfach verwandt werden, bleiben uns nur zwei mögliche Schlußfolgerungen :

- entweder ist unsere Kritik richtig und die Resultate dieser Anwendungen sind wissenschaftlich nicht brauchbar,

oder

- mit diesem Verfahren werden tatsächlich kausale Zusammenhänge gefunden und dann ist unsere Kritik unangemessen.

Eine mögliche Kritik an unserer Position könnte auch in der verwunderten Frage bestehen, ob wir denn tatsächlich behaupten wollen, daß alle jemals von Soziologen mit Kausalanalysemethoden gefundenen Zusammenhänge in Wirklichkeit gar nicht existierten? Wird die Astrologie dadurch zur Wissenschaft, da B einige ihrer Prognosen tatsächlich eingetroffen sind?

Wer die Literatur nach inhaltlich plausiblen kausalen Gesetzmäßigkeiten durchforstet und dabei glaubt, fündig geworden zu sein, hat dadurch noch kein Argument gegen die hier vorgetragene Kritik gefunden, denn:

- Zum einen werden nur die Resultate von Analysen publiziert, die inhaltlich plausibel sind. Durch diesen bei Signifikanztests hinreichend gut bekannten Automatismus werden methodisch ungenügende Verfahren sicher vor einer praktischen Widerlegung geschützt. Im Prinzip ist dies nichts 
anderes als der Verkauf von Glückszahlen im Lotto mit den in der Werbung abgedruckten Dankesbriefen.

- Zum anderen ist selbst bei den vergleichsweise unflexiblen Modellen für die nominalskalierte Datenanalyse die Menge der möglichen Modelle in einer Anwendung noch immer hoch genug, um eine gute Zufallschance für ein inhaltlich plausibles Resultat zu ergeben.

Überdies sollten zwei Tatsachen nicht übersehen werden:

- Der massive Einsatz von EDV in der empirischen Sozialwissenschaft und die damit verbundene Verbreitung von Kausalanalyseprogrammen hat eben nicht zu einem Fundus an kausalem soziologischen Wissen geführt. Unser Wissen auf diesem Gebiet ist noch immer durch Alltagskompetenzen erworben und nicht durch die in der soziologischen Kausalanalyse verwendeten Verfahren. Und überdies darf bezweifelt werden, daB es sich dabei um ein Wissen über kausale Zusammenhänge handelt. Das Ausbleiben eines durch die Verbreitung der einschlägigen Verfahren bewirkten Kumulationseffektes dürfte das schärfste praktische Argument gegen diese Verfahren überhaupt sein.

- Wenn Soziologen von der Kausalanalyse reden, dann geschieht dies oft in einer so lockeren Weise, daß ein weiter Spielraum für die Interpretation der praktisch erzielten Resultate bleibt. Sehr deutlich wird dies u.a. bei der Deutung der als unabhängig gedachten Ursachen in einem konkreten Modell. Es ist eine seltene Ausnahme, wenn ein Autor deutlich macht, ob er eine in die Rechnung einbezogene potentielle Ursache als hinreichend für die Herbeiführung eines Effektes versteht, oder ob sie in einem (interpretatorisch nicht weiter erläuterten) Zusammenhang mit anderen Variablen/Interaktionen zu dem Effekt führt. Sehr viele der in Veröffentlichungen genannten Ursachen sind vermutet, allenfalls im Zusammenhang mit anderen nicht berücksichtigten Variablen kausal relevant, wobei allerdings ungeklärt bleibt, wie sich der mitgeteilte Zusammenhang dadurch verändern würde. Was aus solchen Arbeiten hervorgeht, sind nicht präzise Kausalanalysen, sondern bestenfalls Segmente aus einem größeren kausalen $\mathrm{Zu}$ sammenhang, wobei die Auswahlgesichtspunkte dunkel bleiben.

Jedenfalls liefern die mit Analyseverfahren erzielten praktischen Resultate keine Argumente, die unsere theoretische Kritik erschüttern könnten. Imı Gegenteil: Der theoretisch zu erwartende Effekt, daß nämlich eine Kumulation kausalen Wissens nicht stattfinden kann, läßt sich durch eine Analyse der praktischen Anwendungen bestätigen. Es gibt keine in der Anwendung dieser Verfahren sichtbar werdende praktische Vernunft, die der theoretischen Kritik ihre Spitze nehmen könnte. Der mit der Anwendung dieser Verfahren notwendig erhobene Anspruch auf die Explikation eines kausalen Wissens in soziologischen Anwendungsbereichen wird nicht eingelöst, weil 
er gar nicht eingelöst werden kann. Die Praxis ist so defizitär, wie wir es von unseren theoretischen Analysen her erwarten konnten und darf deswegen als eine Bestätigung der explizierten Defizite angesehen werden.

Wie viele der methodologisch orientierten theoretischen Kritiken wird auch diese folgenlos bleiben. Soziologen werden fortfahren Daten zu sammeln, kausale Modelle zu entwerfen, die Übereinstimmung dieser Modelle mit den Daten zu prüfen und beim Gelingen der Tests entsprechende Resultate verkünden. Vermutlich würde mancher bestimmte Defizite der Analysetechniken einräumen und zugleich die heuristische Nüzlichkeit der Verfahren betonen. Aber wie lange darf man mit der wissenschaftlichen Hoffnung leben, eine defizitäre Heuristik würde schon eines Tages in eine wissenschaftliche Systematik übergehen, ohne an Glaubwürdigkeit zu verlieren? Für soziologische Kausalanalytiker scheint es ein rational abschätzbares zeitliches Limit nicht zu geben, so daß die nun zitierten Auszüge aus dem Urteil des Jahres 2043 wohl niemals ihre Gültigkeit einbüßen werden:

"...Wir sehen zunächst ganz von dem Anspruch der Angeklagten ab, mit ihren Methoden nicht-experimentelle naturwissenschaftliche Kausalanalysen betreiben zu wollen und beziehen uns nur auf die Anwendung der Methoden in ihrem ureigensten Gegenstandsbereich. Selbst unter hochgradig idealisierten Bedingungen kann von den kausalanalytischen Verfahren der Soziologie eine Entdeckung kausaler Gesetze nicht erwartet werden. Es ist schlicht unvorstellbar, wie mit den von den Angeklagten verwendeten Methoden eine Kumulation kausalen Wissens stattfinden soll.

Für diese Möglichkeit liefert weder die Anwendung dieser Verfahren vor mehr als so Jahren noch ihre theoretische Analyse ein Indiz. Die methodologische Oberflächlichkeit, mit der diese Verfahren verwendet wurden, ist erschreckend. Ihre Insuffizienz für die Erreichung der intendierten Forschungsziele hätte bei einer auch nur halbwegs sorgfältigen Analyse ohne weiteres erkannt werden können.

$\mathrm{Da} B$ die Angeklagten nunmehr versucht haben, nicht-experimentelle Methoden auf naturwissenschaftliche Problemfelder anzuwenden, ist verständlich. Denn schließlich waren es ja gerade die als ungemein erfolgreich angesehenen Naturwissenschaften, die die Entwicklung kausaler Analysen in den Sozialwissenschaften stimulierten. Unverständlich bleibt, warum von den Propagandisten dieser Entwicklung nie die gewaltigen strukturellen Differenzen zwischen

- kontrollierten Experimenten

und

- holistischen Entwicklungen, die sich einer experimentellen Systematisierung entziehen,

gesehen wurden. Auch in der naturwissenschaftlichen Forschung waren multivariate holistische Vorgänge nur in solchen Teilbereichen durch kausale Gesetze zu erklären, wo man sich auf ein entsprechendes experimentell gewonnenes Detailwissen stützen konnte. Die Schwierigkeiten von Meteorologen, auch mit größtem Forschungsaufwand zu guten einwöchigen Prognosen zu gelangen, sind dafür ein Beleg von vielen.

Soziologische Kausalanalyse, deren Problemfelder durch Schlagworte wie 'holistisch' und 'in extremer Weise multivariat' zutreffend gekennzeichnet 
sind, hätte sich allenfalls an entsprechenden holistischen, nicht experimentell zugänglichen naturwissenschaftlichen Forschungsgebieten orientieren können. 'Orientieren können' soll hier vor allem auf die Möglichkeit verweisen, die immensen Schwierigkeiten zu studieren, die einer kausalgesetzlichen Strukturierung des Gegenstandsbereiches entgegenstehen...,

... bleibt uns nur die zwingende Schlußfolgerung: Mit den vorgeschlagenen Forschungsmethoden konnte es eine kausale Analyse in der Soziologie nicht geben. Was in diesem Fall getan wurde, war wissenschaftlich schlicht wertlos ..." 